\title{
FINDING LOW-RANK SOLUTIONS OF SPARSE LINEAR MATRIX INEQUALITIES USING CONVEX OPTIMIZATION
}

\author{
RAMTIN MADANI, SOMAYEH SOJOUDI, GHAZAL FAZELNIA AND JAVAD LAVAEI *
}

\begin{abstract}
This paper is concerned with the problem of finding a low-rank solution of an arbitrary sparse linear matrix inequality (LMI). To this end, we map the sparsity of the LMI problem into a graph. We develop a mathematical framework to relate the rank of the minimum-rank solution of the LMI problem to the sparsity of its underlying graph. Furthermore, we propose three graphtheoretic convex programs to obtain a low-rank solution. Two of these convex optimization problems are based on a tree decomposition of the sparsity graph. The third one does not rely on any computationally-expensive graph analysis and is always polynomial-time solvable, at the cost of offering a milder theoretical guarantee on the rank of the obtained solution compared to the other two methods. The results of this work can be readily applied to three separate problems of minimumrank matrix completion, conic relaxation for polynomial optimization, and affine rank minimization. The results are finally illustrated on two applications of optimal distributed control and nonlinear optimization for electrical networks.
\end{abstract}

1. Introduction. Let $\mathbb{S}_{n}$ denote the set of $n \times n$ real symmetric matrices and $\mathbb{S}_{n}^{+}$denote the cone of positive semidefinite matrices in $\mathbb{S}_{n}$. Consider the linear matrix inequality (LMI) problem

$$
\begin{array}{ll}
\text { find } & \mathbf{X} \in \mathbb{S}_{n} \\
\text { subject to } & \operatorname{trace}\left\{\mathbf{M}_{k} \mathbf{X}\right\} \leq a_{k}, \quad k=1, \ldots, p, \\
& \mathbf{X} \succeq 0,
\end{array}
$$

where $\succeq$ represents the positive semidefinite sign, $\mathbf{M}_{1}, \ldots, \mathbf{M}_{p} \in \mathbb{S}_{n}$ are sparse matrices and $a_{1}, \ldots, a_{p} \in \mathbb{R}$ are arbitrary fixed scalars. The formulation given in (1.1) includes problems with equality constraints, but requires rewriting each equality as two inequality constraints. The objective of this paper is twofold. First, it is aimed to find a low-rank solution $\mathbf{X}^{\mathrm{opt}}$ of the above LMI feasibility problem using a convex program. Second, it is intended to study the relationship between the rank of such a low-rank solution and the sparsity level of the matrices $\mathbf{M}_{1}, \ldots, \mathbf{M}_{k}$. To formulate the problem, let $P \subseteq \mathbb{S}_{n}$ denote the convex polytope characterized by the linear inequalities given in (1.1a). In this work, the goal is to design an efficient algorithm to identify a low-rank matrix $\mathbf{X}^{\text {opt }}$ in the set $\mathbb{S}_{n}^{+} \cap P$.

Finding any feasible solution for (1.1) amounts to a semidefinite program, which under mild technical assumptions can be solved within $\varepsilon$-approximation in polynomial time (in terms of the problem size and $\log \left(\varepsilon^{-1}\right)$ ) 1]. However, since commonly-used numerical algorithms (namely interior-point methods) tend to obtain a highest-rank

${ }^{*}$ Ramtin Madani is with the Electrical Engineering Department at the University of Texas at Arlington (ramtin.madani@uta.edu). Somayeh Sojoudi is with the Department of Industrial Engineering and Operations Research, University of California, Berkeley (sojoudi@berkeley.edu). Ghazal Fazelnia is with the Electrical Engineering Department, Columbia University (gf2293@columbia.edu). Javad Lavaei is with the Department of Industrial Engineering and Operations Research, University of California, Berkeley (lavaei@berkeley.edu). This work was supported by a Google Research Award, NSF CAREER Award, ONR YIP Award, DARPA YFA Award, and AFOSR YIP Award. Parts of this work have appeared in the conference paper: "Ramtin Madani, Ghazal Fazelnia, Somayeh Sojoudi and Javad Lavaei, Low-Rank Solutions of Matrix Inequalities With Applications to Polynomial Optimization and Matrix Completion Problems, IEEE Conference on Decision and Control, 2014". 
feasible point, the problem of finding low-rank solutions for 1.1) requires further studies. The special case where $P$ is an affine subspace of $\mathbb{S}_{n}$ (i.e., it is characterized by linear equality constraints) has been extensively studied in the literature [2, 3, 4]. In particular, the work [3] derives an upper bound on the rank of $\mathbf{X}^{\mathrm{opt}}$, which depends on the dimension of $P$ as opposed to the sparsity level of the problem. The paper [4] develops a polynomial-time algorithm to find a solution satisfying the bound condition given in [3]. However, since the bound obtained in [3] is independent of the sparsity of the LMI problem (1.1), it is known not to be tight for several practical examples [5, 6].

The investigation of low-rank solutions for the above-mentioned LMI has direct applications in three fundamental problems: (i) minimum-rank positive semidefinite matrix completion, (ii) conic relaxation for polynomial optimization, and (iii) affine rank minimization. In what follows, these problems will be introduced in three separate subsections, followed by an outline of our contribution for each problem.

1.1. Low-rank Positive Semidefinite Matrix Completion. The LMI problem (1.1) encapsulates the low-rank positive semidefinite matrix completion problem, which is as follows: given a partially completed matrix with some known entries, the positive semidefinite matrix completion problem aims to design the unknown (free) entries of the matrix in such a way that the completed matrix becomes positive semidefinite. As a classical result, this problem has been fully addressed in [7, provided the graph capturing the locations of the known entries of the matrix is chordal. The positive semidefinite matrix completion problem plays a critical role in reducing the complexity of large-scale semidefinite programs [8, 9, 10, 11, 12, 13. In the case where a minimum-rank completion is sought, the problem is referred to as minimumrank positive semidefinite matrix completion. To formalize this problem, consider a simple graph $\mathcal{G}=\left(\mathcal{V}_{\mathcal{G}}, \mathcal{E}_{\mathcal{G}}\right)$ with the vertex set $\mathcal{V}_{\mathcal{G}}$ and the edge set $\mathcal{E}_{\mathcal{G}}$. Let $\operatorname{gd}(\mathcal{G})$ denote the Gram dimension of $\mathcal{G}$, defined as the smallest positive integer $r$ such that the feasibility problem

$$
\begin{array}{llr}
\text { find } & \mathbf{X} \in \mathbb{S}_{|\mathcal{G}|} \\
\text { subject to } & X_{i j}=\widehat{X}_{i j}, \quad(i, j) \in \mathcal{E}_{\mathcal{G}}, \\
& X_{k k}=\widehat{X}_{k k}, \quad k \in \mathcal{V}_{\mathcal{G}}, \\
& \mathbf{X} \succeq 0,
\end{array}
$$

has a solution with rank less than or equal to $r$ for every $\widehat{\mathbf{X}} \in \mathbb{S}_{|\mathcal{G}|}^{+}$, where $|\mathcal{G}|$ denotes the number vertices of $\mathcal{G}$. According to the above definition, every arbitrary positive semidefinite matrix $\widehat{\mathbf{X}}$ can be turned into a matrix $\mathbf{X}$ with rank at most $\operatorname{gd}(\mathcal{G})$ by manipulating those off-diagonal entries of $\widehat{\mathbf{X}}$ that correspond to the non-existent edges of $\mathcal{G}$. The paper [14] introduces the notion of Gram dimension and shows that $\operatorname{gd}(\mathcal{G}) \leq$ $\operatorname{tw}(\mathcal{G})+1$ (for real-valued problems), where $\operatorname{tw}(\mathcal{G})$ denotes the treewidth of the graph $\mathcal{G}$.

There is a large body of literature on two graph-theoretic parameters about the minimum semidefinite rank of a graph over the space of real symmetric or complex Hermitian matrices [15, 16, 17]. These two parameters, denoted as $\operatorname{msr}_{\mathbb{S}}(\mathcal{G})$ and $\operatorname{msr}_{\mathbb{H}}(\mathcal{G})$, are respectively equal to the smallest ranks of all positive semidefinite matrices in $\mathbb{S}$ and $\mathbb{H}$ whose off-diagonal parts have the same support as the adjacency matrix of $\mathcal{G}$. It is straightforward to verfiy that $\operatorname{msr}_{\mathbb{H}}(\mathcal{G})$ is a lower bound for $\operatorname{msr}_{\mathbb{S}}(\mathcal{G})$. In [18, a simple graph $\mathcal{G}$ is provided for the first time with the property $\operatorname{msr}_{\mathbb{H}}(\mathcal{G})<\operatorname{msr}_{\mathbb{S}}(\mathcal{G})$. The notion of OS-vertex number of $\mathcal{G}$, denoted by $\mathrm{OS}(\mathcal{G})$, is 
proposed in [19] that serves as a lower bound on $\operatorname{msr}_{\mathbb{H}}(\mathcal{G})$. The paper [19] also shows that $\operatorname{OS}(\mathcal{G})=\operatorname{msr}_{\mathbb{H}}(\mathcal{G})$ for every chordal graph $\mathcal{G}$. Some examples of graphs with $\operatorname{OS}(\mathcal{G})<\operatorname{msr}_{\mathbb{H}}(\mathcal{G})$ are also provided in $[20$. The positive semidefinite zero forcing number of a graph $\mathcal{G}$, denoted by $\mathrm{Z}^{+}(\mathcal{G})$, has first been introduced in [18] and used for the computation of msr of certain graphs. It is shown in [18 that

$$
\mathrm{Z}^{+}(\mathcal{G})+\operatorname{OS}(\mathcal{G})=|\mathcal{G}|
$$

for every arbitrary graph $\mathcal{G}$. The reader can refer to [21] and [22] for comprehensive reviews of the relationship between the graph theoretic parameters of $\mathrm{tw}, \mathrm{msr}_{\mathbb{S}}, \mathrm{msr}_{\mathbb{H}}$, OS and $\mathrm{Z}^{+}$.

The matrix completion problem 1.2 can be cast as the LMI problem (1.1) after expressing the constraints $1.2 \mathrm{a}$ and $\left[1.2 \mathrm{~b}\right.$ as trace $\left\{\left(e_{j} e_{i}^{T}+e_{i} e_{j}^{T}\right) \mathbf{X}\right\}=2 \widehat{X}_{i j}$ and trace $\left\{e_{k} e_{k}^{T} \mathbf{X}\right\}=\widehat{X}_{k k}$, where $\left\{e_{1}, \ldots, e_{|\mathcal{G}|}\right\}$ is the standard basis for $\mathbb{R}^{|\mathcal{G}|}$. Hence, the minimum-rank positive semidefinite matrix completion problem can be formulated as finding a minimum-rank matrix in the convex set $\mathbb{S}_{n}^{+} \cap P$. In this work, we utilize the notions of tree decomposition, minimum semidefinite rank of a graph, OS-vertex and positive semidefinite zero forcing to find low-rank matrices in $\mathbb{S}_{n}^{+} \cap P$ using convex optimization. Let $\mathcal{G}$ denote a graph capturing the sparsity of the LMI problem (1.1). Consider the convex problem of minimizing a weighted sum of an arbitrary subset of the free entries of $\mathbf{X}$ subject to the matrix completion constraint of 1.2 . We show that the rank of every solution of this problem can be upper bounded in terms of the OS and msr of some supergraphs of $\mathcal{G}$. Our bound depends only on the locations of the free entries minimized in the objective function rather than their coefficients. In particular, given an arbitrary tree decomposition of $\mathcal{G}$ with width $t$, we show that the minimization of a weighted sum of certain free entries of $\mathbf{X}$ guarantees that every solution $\mathbf{X}^{\text {opt }}$ of this problem belongs to $\mathbb{S}_{n}^{+} \cap P$ and satisfies the relation $\operatorname{rank}\left\{\mathbf{X}^{\mathrm{opt}}\right\} \leq t+1$, for all possible nonzero coefficients of the objective function. This result holds for both real- and complex-valued problems. The problem of finding a tree decomposition of minimum width is NP-complete [23]. Nevertheless, for a fixed integer $t$, the problem of checking the existence of a tree decomposition of width $t$ and finding such a decomposition (if any) can be solved in linear time [24, 25]. Moreover, there are many efficient algorithms in the literature that provide lower and upper bounds on treewidth [26, 27. Whenever a minimal tree decomposition is known, we offer infinitely many optimization problems such that every solution of those problems satisfies the relation $\operatorname{rank}\left\{\mathbf{X}^{\mathrm{opt}}\right\} \leq \mathrm{tw}(\mathcal{G})+1$.

In the case where a good decomposition of $\mathcal{G}$ with small width is not known, we propose a polynomial-time solvable optimization that is able to find a matrix in $\mathbb{S}_{n}^{+} \cap P$ with rank at most $2\left(n-\operatorname{msr}_{\mathbb{H}}(\mathcal{G})\right)$. Note that this solution can be found in polynomial time, whereas our theoretical upper bound on its rank is hard to compute. The upper bound $2\left(n-\operatorname{msr}_{\mathbb{H}}(\mathcal{G})\right)$ is a small number for a wide class of sparse graphs [28.

1.2. Sparse Quadratically-Constrained Quadratic Program. The problem of searching for a low-rank matrix in the convex set $\mathbb{S}_{n}^{+} \cap P$ is important due to its application in obtaining suboptimal solutions of quadratically-constrained quadratic programs (QCQPs). Consider the standard nonconvex QCQP problem

$$
\begin{array}{ll}
\underset{x \in \mathbb{R}^{n-1}}{\operatorname{minimize}} & f_{0}(x) \\
\text { subject to } & f_{k}(x) \leq 0, \quad k=1, \ldots, p,
\end{array}
$$


where $f_{k}(x)=x^{\mathrm{T}} \mathbf{A}_{k} x+2 b_{k}^{\mathrm{T}} x+c_{k}$ for $k=0, \ldots, p$. Every polynomial optimization can be cast as problem (1.4) and this also includes all combinatorial optimization problems [29, 30. Thus, the above nonconvex QCQP "covers almost everything" [30. To tackle this NP-hard problem, define

$$
\mathbf{F}_{k} \triangleq\left[\begin{array}{cc}
c_{k} & b_{k}^{\mathrm{T}} \\
b_{k} & \mathbf{A}_{k}
\end{array}\right] \text {. }
$$

Each $f_{k}$ has the linear representation $f_{k}(x)=\operatorname{trace}\left\{\mathbf{F}_{k} \mathbf{X}\right\}$ for the following choice of $\mathrm{X}$ :

$$
\mathbf{X} \triangleq\left[\begin{array}{ll}
1 & x^{\mathrm{T}}
\end{array}\right]^{\mathrm{T}}\left[\begin{array}{ll}
1 & x^{\mathrm{T}}
\end{array}\right]
$$

It is obvious that an arbitrary matrix $\mathbf{X} \in \mathbb{S}_{n}$ can be factorized as 1.6 if and only if it satisfies the three properties $X_{11}=1, \mathbf{X} \succeq 0$, and $\operatorname{rank}\{\mathbf{X}\}=1$. Therefore, problem (1.4) can be reformulated as follows:

$$
\begin{array}{ll}
\underset{\mathbf{X} \in \mathbb{S}_{n}}{\operatorname{minimize}} & \operatorname{trace}\left\{\mathbf{F}_{0} \mathbf{X}\right\} \\
\text { subject to } & \operatorname{trace}\left\{\mathbf{F}_{k} \mathbf{X}\right\} \leq 0 \quad k=1, \ldots, p, \\
& X_{11}=1, \\
& \mathbf{X} \succeq 0, \\
& \operatorname{rank}\{\mathbf{X}\}=1 .
\end{array}
$$

In the above representation of QCQP, the constraint $1.7 \mathrm{e}$ carries all the nonconvexity. Neglecting this constraint yields a convex problem, known as the semidefinite programming (SDP) relaxation of QCQP [31, 32. The existence of a rank-1 solution for the SDP relaxation guarantees the equivalence of the original QCQP and its relaxed problem.

The SDP relaxation technique provides a lower bound on the minimum cost of the original problem, which can be used for various purposes such as the branch and bound algorithm [30. To understand the quality of the SDP relaxation, this lower bound is known to be at most $14 \%$ less than the minimum cost for the MAXCUT problem [33. In general, the maximum possible gap between the solution of a graph optimization and that of its SDP relaxation is defined as the Grothendieck constant of the graph [34, 35. This constant is calculated for some special graphs in 36. If the QCQP problem and its SDP relaxation result in the same optimal objective value, then the relaxation is said to be exact. The exactness of the relaxation is substantiated for various applications [37, 38, 39, 40.

By exploring the optimal power flow problem, we have shown in 41 that the exactness of the relaxation could be heavily formulation dependent. Indeed, we have designed a practical circuit optimization with four equivalent QCQP formulations, where only one of them has an exact SDP relaxation. In the same context, we have also verified in [41] that the SDP relaxation may have a hidden rank-1 solution that could not be easily found. The reason is that the SDP relaxation of a sparse QCQP problem often has infinitely many solutions and the conventional numerical algorithms would find a solution with the highest rank. Hence, a question arises as to whether a low-rank solution of the SDP relaxation of a sparse QCQP can be found efficiently. To address this problem, let $\widehat{\mathbf{X}}$ denote an arbitrary solution of the SDP relaxation. If the QCQP problem (1.4) is sparse and associated with a sparsity graph $\mathcal{G}$, then every positive semidefinite matrix $\mathbf{X}$ satisfying the matrix completion constraint 1.2 is another 
solution of the SDP relaxation of the QCQP problem. Using this matrix completion technique, the results spelled out in the preceding subsection can be exploited to find a low-rank SDP solution.

1.3. Affine Rank Minimization Problem. Consider the problem

$$
\begin{array}{ll}
\underset{\mathbf{W} \in \mathbb{R}^{m \times r}}{\operatorname{minimize}} & \operatorname{rank}\{\mathbf{W}\} \\
\text { subject to } & \operatorname{trace}\left\{\mathbf{N}_{k} \mathbf{W}\right\} \leq a_{k}, \quad k=1, \ldots, p,
\end{array}
$$

where $\mathbf{N}_{1}, \ldots, \mathbf{N}_{p} \in \mathbb{R}^{r \times m}$ are sparse matrices. This is an affine rank minimization problem without any positive semidefinite constraint. A popular convexification method for the above non-convex optimization is to replace its objective with the nuclear norm of $\mathbf{W}$ [42. This is due to the fact that the nuclear norm $\|\mathbf{W}\|_{*}$ is the convex envelop for the function $\operatorname{rank}\{\mathbf{W}\}$ on the set $\left\{\mathbf{W} \in \mathbb{R}^{m \times r} \mid\|\mathbf{W}\| \leq 1\right\}$ [43. A special case of Optimization (1.8), known as low-rank matrix completion problem, has been extensively studied in the literature due to its wide applications [44, 45, 42, 46]. In this problem, the constraint 1.8 determines what entries of $\mathbf{W}$ are known.

A closely related problem is the following: can a matrix $\mathbf{W}$ be recovered by observing only a subset of its entries? Interestingly, $\mathbf{W}$ can be successfully recovered by means of a nuclear norm minimization as long as the matrix is non-structured and the number of observed entries of $\mathbf{W}$ is large enough [45, 47, 46. The performance of the nuclear norm minimization method for the problem of rank minimization subject to general linear constraints has also been assessed in [48. Based on empirical studies, the nuclear norm technique is inefficient in the case where the number of free (unconstrained) entries of $\mathbf{W}$ is relatively large. In the present work, we propose a graph-theoretic approach that is able to generate low-rank solutions for a sparse problem of the form (1.8) and for a matrix completion problem with many unknown entries.

Optimization 1.8 can be embedded in a bigger problem of the form 1.1 by associating the matrix $\mathbf{W}$ with a positive semidefinite matrix variable $\mathbf{X}$ defined as

$$
\mathbf{X} \triangleq\left[\begin{array}{cc}
\mathbf{X}_{1} & \mathbf{W} \\
\mathbf{W}^{\mathrm{T}} & \mathbf{X}_{2}
\end{array}\right]
$$

where $\mathbf{X}_{1}$ and $\mathbf{X}_{2}$ are two auxiliary matrices. Note that $\mathbf{W}$ acts as a submatrix of $\mathbf{X}$ corresponding to its first $m$ rows and last $r$ columns. More precisely, consider the nonconvex problem

$$
\begin{array}{ll}
\underset{\mathbf{X} \in \mathbb{S}_{r+m}}{\operatorname{minimize}} & \operatorname{rank}\{\mathbf{X}\} \\
\text { subject to } & \operatorname{trace}\left\{\mathbf{M}_{k} \mathbf{X}\right\} \leq a_{k}, \quad k=1, \ldots, p, \\
& \mathbf{X} \succeq 0,
\end{array}
$$

where

$$
\mathbf{M}_{k} \triangleq\left[\begin{array}{cc}
0_{m \times m} & \frac{1}{2} \mathbf{N}_{k}^{\mathrm{T}} \\
\frac{1}{2} \mathbf{N}_{k} & 0_{r \times r}
\end{array}\right] .
$$

For every feasible solution $\mathbf{X}$ of the above problem, its associated submatrix $\mathbf{W}$ is feasible for (1.8) and satisfies

$$
\operatorname{rank}\{\mathbf{W}\} \leq \operatorname{rank}\{\mathbf{X}\}
$$


In particular, it is well known that the rank minimization problem 1.8 with linear constraints is equivalent to the rank minimization (1.10) with LMI constraints [43, 49]. Let $\widehat{\mathbf{X}}$ denote an arbitrary feasible point of optimization 1.10 . Depending on the sparsity level of the problem 1.8, some entries of $\widehat{\mathbf{X}}$ are free and do not affect any constraints of 1.10 except for $\mathbf{X} \succeq 0$. Let the locations of those entries be captured by a bipartite graph. More precisely, define $\mathcal{B}$ as a bipartite graph whose first and second parts of vertices are associated with the rows and columns of $\mathbf{W}$, respectively. Suppose that each edge of $\mathcal{B}$ represents a constrained entry of $\mathbf{W}$. In this work, we propose two convex problems with the following properties:

1. The first convex program is constructed from an arbitrary tree decomposition of $\mathcal{B}$. The rank of every solution to this problem is upper bounded by $t+1$, where $t$ is the width of its tree decomposition. Given the decomposition, the low-rank solution can be found in polynomial time.

2. Since finding a tree decomposition of $\mathcal{B}$ with a low treewidth may be hard in general, the second convex program does not rely on any decomposition and is obtained by relaxing the real-valued problem (1.10) to a complex-valued convex program. The rank of every solution to the second convex problem is bounded by the number $2\left(r+m-\operatorname{msr}_{\mathbb{H}}\{\mathcal{B}\}\right)$ and such a solution can always be found in polynomial time.

1.4. Simple Illustrative Examples. To illustrate some of the main ideas to be discussed in this work, three simple examples will be provided below in the context of low-rank positive semidefinite matrix completion.

EXAmple 1. Consider a partially-known matrix $\mathbf{X} \in \mathbb{S}_{n}^{+}$with unknown offdiagonal entries and known strictly positive diagonal entries $X_{11}, \ldots, X_{n n}$. The aim is to design the unknown off-diagonal entries of $\mathbf{X}$ to make the resulting matrix as low rank as possible. It can be shown that there are $2^{n}$ rank-1 matrices $\mathbf{X} \in \mathbb{S}_{n}^{+}$with the diagonal entries $X_{11}, \ldots, X_{n n}$, each of which can be expressed as $x x^{\mathrm{T}}$ for a vector $x$ with the property that $x_{i}= \pm \sqrt{X_{i i}}$. A question arises as to whether such matrix completions can be attained via solving a convex optimization. To address this question, consider the problem of finding a matrix $\mathbf{X} \in \mathbb{S}_{n}^{+}$with the given diagonal to minimize an arbitrary weighted sum of the subdiagonal entries of $\mathbf{X}$, i.e., $\sum_{i=1}^{n-1} t_{i} X_{i+1, i}$ for arbitrary nonzero coefficients $t_{1}, \ldots, t_{n-1}$. It can be verified that every solution of this optimization problem results in one of the aforementioned $2^{n}$ rank-1 matrices $\mathbf{X}$. In other words, there are $2^{n}$ ways to fill the matrix $\mathbf{X}$, each of which corresponds to infinitely many easy-to-characterize continuous optimization problems.

Example 2. Consider a $3 \times 3$ symmetric block matrix $\mathbf{X}$ partitioned as

$$
\mathbf{X}=\left[\begin{array}{lll}
\mathbf{X}_{11} & \mathbf{X}_{12} & \mathbf{X}_{13} \\
\mathbf{X}_{21} & \mathbf{X}_{22} & \mathbf{X}_{23} \\
\mathbf{X}_{31} & \mathbf{X}_{32} & \mathbf{X}_{33}
\end{array}\right]
$$

where $\mathbf{X}_{11} \in \mathbb{R}^{\alpha \times \alpha}, \mathbf{X}_{22} \in \mathbb{R}^{\beta \times \beta}$ and $\mathbf{X}_{33} \in \mathbb{R}^{\gamma \times \gamma}$, for some positive numbers $\alpha, \beta$ and $\gamma$. Assume that the block $\mathbf{X}_{13}$ is unknown while the remaining blocks of $\mathbf{X}$ are known either partially or completely. Suppose that $\mathbf{X}$ admits a positive definite matrix completion, which implies that $\operatorname{rank}\{\mathbf{X}\} \geq \max \{\alpha+\beta, \beta+\gamma\}$. The goal is to perform the completion of $\mathbf{X}$ via convex optimization such that $\operatorname{rank}\{\mathbf{X}\}=\max \{\alpha+\beta, \beta+\gamma\}$.

Consider first the scenario where $\alpha=\gamma$. Let $\left\{\left(i_{1}, j_{1}\right), \ldots,\left(i_{s}, j_{s}\right)\right\}$ denote an arbitrary set of entries of $\mathbf{X}_{13}$ with $s$ elements. Consider the optimization problem of 


\begin{tabular}{|c|c|c|c|}
\hline$x$ & $\mathrm{X}_{11}$ & $\mathrm{X}_{12}$ & $X_{13}$ \\
\hline B & $X_{21}$ & $\mathrm{X}_{22}$ & $X_{23}$ \\
\hline & $X_{31}$ & $\mathrm{X}_{32}$ & $X_{33}$ \\
\hline
\end{tabular}

(a)

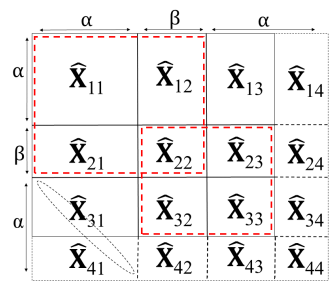

(b)

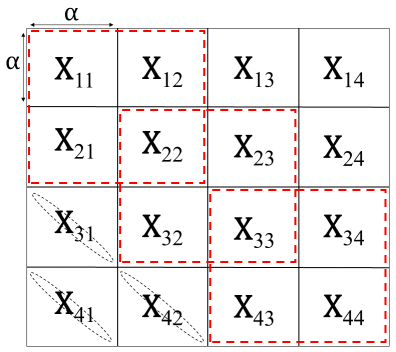

(c)

FIG. 1.1. (a) The matrix $\mathbf{X}$ studied in Example 2 for $\alpha=\gamma$; (b) the augmented matrix $\widehat{\mathbf{X}}$ obtained from $\mathbf{X}$ in the case where $\alpha>\gamma$; (c) the matrix $\mathbf{X}$ studied in Example 3

minimizing $\sum_{k=1}^{s} t_{k} X_{13}\left(i_{k}, j_{k}\right)$ subject to the constraint that $\mathbf{X}$ is a positive semidefinite matrix in the form of (1.13), where $t_{1}, \ldots, t_{s}$ are nonzero scalars and $X_{13}\left(i_{k}, j_{k}\right)$ denotes the $\left(i_{s}, j_{s}\right)$ entry of $\mathbf{X}_{13}$. Let $\mathbf{X}^{\text {opt }}$ be an arbitrary solution of this problem. In this work, we derive an upper bound on the rank of $\mathbf{X}^{\text {opt }}$, which depends only on the set $\left\{\left(i_{1}, j_{1}\right), \ldots,\left(i_{s}, j_{s}\right)\right\}$ and is independent of $t_{1}, \ldots, t_{s}$. In particular, if $\left\{\left(i_{1}, j_{1}\right), \ldots,\left(i_{s}, j_{s}\right)\right\}$ corresponds to $s=\alpha$ entries of $\mathbf{X}_{13}$ with no two elements in the same row or column, then it is guaranteed that $\operatorname{rank}\left\{\mathbf{X}^{\mathrm{opt}}\right\}=\max \{\alpha+\beta, \beta+\gamma\}$ for all nonzero values of $t_{1}, t_{2}, \ldots, t_{s}$. Figure 1.1(a) shows the blocks of matrix $\mathbf{X}$, where the two $2 \times 2$ blocks of $\mathbf{X}$ specified by dashed red lines are known while the block $\mathbf{X}_{31}$ is to be designed. As a special case of the above method, minimizing a weighted sum of the diagonal entries of $\mathbf{X}_{31}$ with nonzero weights leads to a lowest-rank completion.

Consider now the scenario where $\alpha>\gamma$. We add $\alpha-\gamma$ rows and $\alpha-\gamma$ columns to $\mathbf{X}$ and denote the augmented matrix as $\widehat{\mathbf{X}}$. This procedure is demonstrated in Figure $1.1(\mathrm{~b})$, where the added blocks are labeled as $\widehat{\mathbf{X}}_{14}, \widehat{\mathbf{X}}_{24}, \widehat{\mathbf{X}}_{34}, \widehat{\mathbf{X}}_{41}, \widehat{\mathbf{X}}_{42}, \widehat{\mathbf{X}}_{43}$ and $\widehat{\mathbf{X}}_{44}$. Note that the first $\alpha+\beta+\gamma$ rows and $\alpha+\beta+\gamma$ columns of $\widehat{\mathbf{X}}$ are exactly the same as those of the matrix $\mathbf{X}$. We also set all diagonal entries of $\widehat{\mathbf{X}}_{44}$ to 1 . The matrix $\widehat{\mathbf{X}}$ has two partially-known $2 \times 2$ blocks of size $\alpha+\beta$ as well as a square nonoverlapping block containing $\widehat{\mathbf{X}}_{31}$ and $\widehat{\mathbf{X}}_{41}$. The problem under study now reduces to the matrix completion posed in the previous scenario $\alpha=\gamma$. More precisely, consider the problem of minimizing an arbitrary weighted sum of the diagonal entries of the non-overlapping block $\left(\widehat{\mathbf{X}}_{31}, \widehat{\mathbf{X}}_{41}\right)$ with nonzero weights over all positive semidefinite partially-known matrices $\widehat{\mathbf{X}}$. Every solution $\widehat{\mathbf{X}}^{\text {opt }}$ of this optimization has rank at most $\alpha+\beta$, and so does its submatrix $\mathbf{X}^{\text {opt }}$.

Example 3 . Consider the $4 \times 4$ symmetric block matrix $\mathbf{X}$ shown in Figure 1.1(c) with partially-known blocks $\mathbf{X}_{11}, \mathbf{X}_{21}, \mathbf{X}_{22}, \mathbf{X}_{32}, \mathbf{X}_{33}, \mathbf{X}_{43}, \mathbf{X}_{44}$ and totally-unknown blocks $\mathbf{X}_{31}, \mathbf{X}_{41}, \mathbf{X}_{42}$. The goal is to fill the matrix to a minimum-rank positive semidefinite matrix. For simplicity, assume that the matrix $\mathbf{X}$ admits a positive definite completion and that all 16 blocks $\mathbf{X}_{i j}$ have the same size $\alpha \times \alpha$. It can be verified that the matrix $\mathbf{X}$ admits a positive semidefinite completion with rank $2 \alpha$. To convert the problem into an optimization, one can minimize the weighted sum of certain entries of $\mathbf{X}_{31}, \mathbf{X}_{41}, \mathbf{X}_{42}$. It turns that if the weighted sum of the diagonal entries of one or all of these three blocks is minimized, the rank would be higher than $2 \alpha$. However, the minimization of the diagonal entries of the two blocks $\mathbf{X}_{31}$ and $\mathbf{X}_{42}$ always produces a lowest-rank solution. 



FIG. 2.1. A maximal OS-vertex sequence for the Petersen graph

2. Notations and Definitions. The symbols $\mathbb{R}$ and $\mathbb{C}$ denote the sets of real and complex numbers, respectively. $\mathbb{S}_{n}$ denotes the space of $n \times n$ real symmetric matrices and $\mathbb{H}_{n}$ denotes the space of $n \times n$ complex Hermitian matrices. Also, $\mathbb{S}_{n}^{+} \subset \mathbb{S}_{n}$ and $\mathbb{H}_{n}^{+} \subset \mathbb{H}_{n}$ represent the convex cones of real and complex positive semidefinite matrices, respectively. The set of notations $\left(\mathbb{F}_{n}, \mathbb{F}_{n}^{+}, \mathbb{F}\right)$ refers to either $\left(\mathbb{S}_{n}, \mathbb{S}_{n}^{+}, \mathbb{R}\right)$ or $\left(\mathbb{H}_{n}, \mathbb{H}_{n}^{+}, \mathbb{C}\right)$ depending on the context (i.e., whether the real or complex domain is under study). $\operatorname{Re}\{\cdot\}, \operatorname{Im}\{\cdot\}, \operatorname{rank}\{\cdot\}$, and trace $\{\cdot\}$ denote the real part, imaginary part, rank, and trace of a given scalar/matrix. Matrices are shown by capital and bold letters. The symbols $(\cdot)^{\mathrm{T}}$ and $(\cdot)^{*}$ denote transpose and conjugate transpose, respectively. Also, "i" is reserved to denote the imaginary unit. The notation $\measuredangle x$ denotes the angle of a complex number $x$. The notation $\mathbf{W} \succeq 0$ means that $\mathbf{W}$ is a Hermitian and positive semidefinite matrix. The $(i, j)$ entry of $\mathbf{W}$ is denoted as $W_{i j}$, unless otherwise mentioned. Given scalars $x_{1}, \ldots, x_{n}$, the notation $\operatorname{diag}\left\{\left[x_{1}, \ldots, x_{n}\right]\right\}$ denotes a $n \times n$ diagonal matrix with the diagonal entries $x_{1}, \ldots, x_{n}$. The vertex set and edge set of a simple undirected graph $\mathcal{G}$ are shown by the notations $\mathcal{V}_{\mathcal{G}}$ and $\mathcal{E}_{\mathcal{G}}$, and the graph $\mathcal{G}$ is identified by the pair $\left(\mathcal{V}_{\mathcal{G}}, \mathcal{E}_{\mathcal{G}}\right) . \mathcal{N}_{\mathcal{G}}(k)$ denotes the set of all neighbors of the vertex $k$ in the graph $\mathcal{G}$. The symbol $|\mathcal{G}|$ shows the number of vertices of $\mathcal{G}$.

Definition 2.1. For two simple graphs $\mathcal{G}_{1}=\left(\mathcal{V}_{1}, \mathcal{E}_{1}\right)$ and $\mathcal{G}_{2}=\left(\mathcal{V}_{2}, \mathcal{E}_{2}\right)$, the notation $\mathcal{G}_{1} \subseteq \mathcal{G}_{2}$ means that $\mathcal{V}_{1} \subseteq \mathcal{V}_{2}$ and $\mathcal{E}_{1} \subseteq \mathcal{E}_{2}$. $\mathcal{G}_{1}$ is called a subgraph of $\mathcal{G}_{2}$ and $\mathcal{G}_{2}$ is called a supergraph of $\mathcal{G}_{1}$. A subgraph $\mathcal{G}_{1}$ of $\mathcal{G}_{2}$ is said to be an induced subgraph if for every pair of vertices $v_{l}, v_{m} \in \mathcal{V}_{1}$, the relation $\left(v_{l}, v_{m}\right) \in \mathcal{E}_{1}$ holds if and only if $\left(v_{l}, v_{m}\right) \in \mathcal{E}_{2}$. In this case, $\mathcal{G}_{1}$ is said to be induced by the vertex subset $\mathcal{V}_{1}$.

Definition 2.2. For two simple graphs $\mathcal{G}_{1}=\left(\mathcal{V}_{1}, \mathcal{E}_{1}\right)$ and $\mathcal{G}_{2}=\left(\mathcal{V}_{2}, \mathcal{E}_{2}\right)$, the subgraph of $\mathcal{G}_{2}$ induced by the vertex set $\mathcal{V}_{2} \backslash \mathcal{V}_{1}$ is shown by the notation $\mathcal{G}_{2} \backslash \mathcal{G}_{1}$.

Definition 2.3. For two simple graphs $\mathcal{G}_{1}=\left(\mathcal{V}, \mathcal{E}_{1}\right)$ and $\mathcal{G}_{2}=\left(\mathcal{V}, \mathcal{E}_{2}\right)$ with the same set of vertices, their union is defined as $\mathcal{G}_{1} \cup \mathcal{G}_{2}=\left(\mathcal{V}, \mathcal{E}_{1} \cup \mathcal{E}_{2}\right)$ while the notion $\lambda$ shows their subtraction edge-wise, i.e., $\mathcal{G}_{1} \lambda \mathcal{G}_{2}=\left(\mathcal{V}, \mathcal{E}_{1} \backslash \mathcal{E}_{2}\right)$.

DEFINITION 2.4. The representative graph of an $n \times n$ symmetric matrix $\mathbf{W}$, denoted by $\mathscr{G}(\mathbf{W})$, is a simple graph with $n$ vertices whose edges are specified by the locations of the nonzero off-diagonal entries of $\mathbf{W}$. In other words, two arbitrary vertices $i$ and $j$ are connected if $W_{i j}$ is nonzero. 

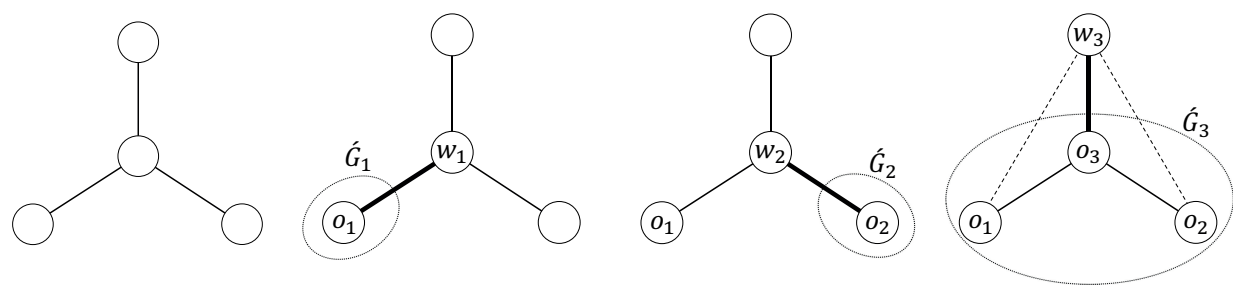

FIG. 2.2. A maximal OS-vertex sequence for a tree
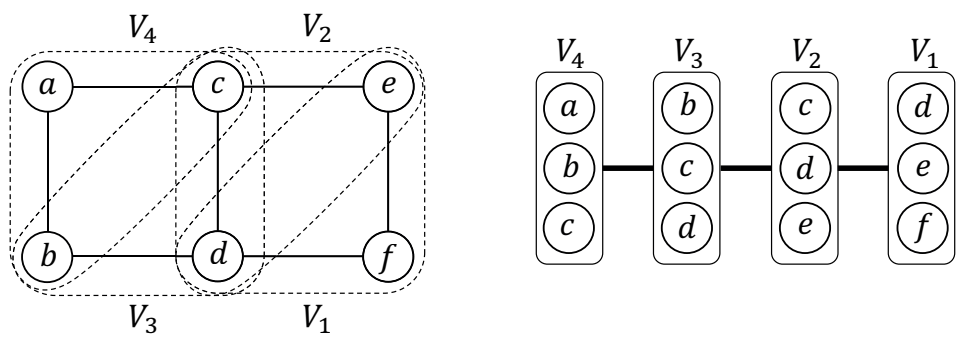

FIG. 2.3. A minimal tree decomposition for a ladder

3. Connection Between OS and Treewidth. In this section, we study the relationship between the graph parameters $O S$ and treewidth. For the sake of completeness, we first review these two graph notions.

DeFINITION $3.1(\mathrm{OS})$. Given a graph $\mathcal{G}$, let $\mathcal{O}=\left\{o_{k}\right\}_{k=1}^{s}$ be a sequence of vertices of $\mathcal{G}$. Define $\mathcal{G}_{k}$ as the subgraph induced by the vertex set $\left\{o_{1}, \ldots, o_{k}\right\}$ for $k=1, \ldots, s$. Let $\mathcal{G}_{k}^{\prime}$ be the connected component of $\mathcal{G}_{k}$ containing $o_{k} . \mathcal{O}$ is called an OS-vertex sequence of $\mathcal{G}$ if for every $k \in\{1, \ldots, s\}$, the vertex $o_{k}$ has a neighbor $w_{k}$ with the following two properties:

1. $w_{k} \neq o_{r}$ for $1 \leq r \leq k$

2. $\left(w_{k}, o_{r}\right) \notin \mathcal{E}_{\mathcal{G}}$ for every $o_{r} \in \mathcal{V}_{G_{k}^{\prime}} \backslash\left\{o_{k}\right\}$,

Denote the maximum cardinality among all OS-vertex sequences of $\mathcal{G}$ as $\operatorname{OS}(\mathcal{G})$ [19] .

Figure 2.1 shows the construction of a maximal OS-vertex sequence for the Petersen graph. Dashed lines and bold lines highlight nonadjacency and adjacency, respectively, to demonstrate that each $w_{i}$ satisfies the conditions of Definition 3.1. Figure 2.2 illustrates the procedure for finding a maximal OS-vertex sequence for a tree. The connected component of each $o_{k}$ in the subgraph induced by $\left\{o_{1}, \ldots, o_{k}\right\}$ is also shown in the picture. Notice that although $w_{2}$ is connected to $o_{1}$, it is a valid choice since $o_{1}$ and $o_{2}$ do not share the same connected component in $\mathcal{G}_{2}$.

Definition 3.2 (Treewidth). Given a graph $\mathcal{G}=\left(\mathcal{V}_{\mathcal{G}}, \mathcal{E}_{\mathcal{G}}\right)$, a tree $\mathcal{T}$ is called a tree decomposition of $\mathcal{G}$ if it satisfies the following properties:

1. Every node of $\mathcal{T}$ corresponds to and is identified by a subset of $\mathcal{V}_{\mathcal{G}}$.

2. Every vertex of $\mathcal{G}$ is a member of at least one node of $\mathcal{T}$.

3. For every edge $(i, j)$ of $\mathcal{G}$, there exists a node in $\mathcal{T}$ containing both vertices $i$ and $j$.

4. Given an arbitrary vertex $k$ of $\mathcal{G}$, the subgraph induced by all nodes of $\mathcal{T}$ containing vertex $k$ must be connected (more precisely, a tree).

Each node of $\mathcal{T}$ is a bag (collection) of vertices of $\mathcal{H}$ and therefore it is referred to as $a$ bag. The width of a tree decomposition is the cardinality of its largest bag minus 
one. The treewidth of $\mathcal{G}$ is the minimum width over all possible tree decompositions of $\mathcal{G}$ and is denoted by $\operatorname{tw}(\mathcal{G})$.

Note that the treewidth of a tree is equal to 1 . Figure 2.3 shows a graph $\mathcal{G}$ with 6 vertices named $a, b, c, d, e, f$, together with a minimal tree decomposition $\mathcal{T}$ with 4 bags $V_{1}, V_{2}, V_{3}, V_{4}$. The width of this decomposition is equal to 2 .

Definition $3.3\left(\mathrm{Z}^{+}\right)$. Let $\mathcal{G}$ be a simple graph. A subset of vertices $\mathcal{Z} \subseteq \mathcal{V}_{\mathcal{G}}$ is called a positive semidefinite zero forcing set of $\mathcal{G}$ if, by starting from $\mathcal{Z}^{\prime}:=\mathcal{Z}$, it is possible to add all of the vertices of $\mathcal{G}$ to $\mathcal{Z}^{\prime}$ by repeating the following operation:

- Choose a vertex $w \in \mathcal{V}_{\mathcal{G}} \backslash \mathcal{Z}^{\prime}$ and let $\mathcal{W}$ be the set of vertices of the connected component of $\mathcal{G} \backslash \mathcal{Z}^{\prime}$ that contains $w$. Add $w$ to $\mathcal{Z}^{\prime}$ if there exists a vertex $u \in \mathcal{Z}^{\prime}$ such that $w$ is the only neighbor of $u$ in the subgraph of $\mathcal{G}$ induced by $\mathcal{W} \cup\{u\}$.

The positive semidefinite zero forcing number of $\mathcal{G}$, denoted by $\mathrm{Z}^{+}(\mathcal{G})$, is the minimum of $|\mathcal{Z}|$ over all positive semidefinite zero forcing sets $\mathcal{Z} \subseteq \mathcal{V}_{\mathcal{G}}$.

DeFINITION 3.4 (Enriched Supergraph). Given a graph $\mathcal{G}$ accompanied by a tree decomposition $\mathcal{T}$ of width $t, \overline{\mathcal{G}}$ is called an enriched supergraph of $\mathcal{G}$ derived by $\mathcal{T}$ if it is obtained according to the following procedure:

1. Add a sufficient number of (redundant) vertices to the bags of $\mathcal{T}$, if necessary, in such a way that every bag includes exactly $t+1$ vertices. Also, add the same vertices to $\mathcal{G}$ (without incorporating new edges). Denote the new graphs associated with $\mathcal{T}$ and $\mathcal{G}$ as $\overline{\mathcal{T}}$ and $\overline{\mathcal{G}}$, respectively. Set $\mathcal{O}$ as the empty sequence and $\tilde{\mathcal{T}}=\overline{\mathcal{T}}$.

2. Identify a leaf of $\tilde{\mathcal{T}}$, named $V$. Let $V^{\prime}$ denote the neighbor of $V$ in $\tilde{\mathcal{T}}$.

3. Let $V \backslash V^{\prime}=\left\{o_{1}, \ldots, o_{s}\right\}$ and $V^{\prime} \backslash V=\left\{w_{1}, \ldots, w_{s}\right\}$. Update $\mathcal{O}, \overline{\mathcal{G}}$ and $\tilde{\mathcal{T}}$ as

$$
\begin{aligned}
& \mathcal{O}:=\mathcal{O} \cup\left\{o_{1}, \ldots, o_{s}\right\} \\
& \overline{\mathcal{G}}:=\left(\mathcal{V}_{\overline{\mathcal{G}}}, \mathcal{E}_{\overline{\mathcal{G}}} \cup\left\{\left(o_{1}, w_{1}\right), \ldots,\left(o_{s}, w_{s}\right)\right\}\right) \\
& \tilde{\mathcal{T}}:=\tilde{\mathcal{T}} \backslash V .
\end{aligned}
$$

4. If $\tilde{\mathcal{T}}$ has more than one bag, go to Step 2. Otherwise, terminate.

The graph $\overline{\mathcal{G}}$ is referred to as an enriched supergraph of $\mathcal{G}$ derived by $\mathcal{T}$. Moreover, $\mathcal{O}$ serves as an OS-vertex sequence for this supergraph and every bag of $\overline{\mathcal{T}}$ is a positive semidefinite zero forcing set for $\overline{\mathcal{G}}$ (see Theorem 3.5).

Figure 3.1(a) illustrates Step 3 of the above definition. Figure 3.2 delineates the process of obtaining an enriched supergraph $\overline{\mathcal{G}}$ of the graph $\mathcal{G}$ depicted in Figure 2.3 . Bold lines show the added edges at each step of the algorithm. Figure 3.1. (b) sketches the resulting OS-vertex sequence $\mathcal{O}$. Observe that whether or not each non-bold edge exists in the graph, $\mathcal{O}$ still remains an OS-vertex sequence. The next theorem reveals the relationship between OS and treewidth.

THEOREM 3.5. Given a graph $\mathcal{G}$ accompanied by a tree decomposition $\mathcal{T}$ of width $t$, consider the enriched supergraph $\overline{\mathcal{G}}$ of $\mathcal{G}$ derived by $\mathcal{T}$ together with the sequence $\mathcal{O}$ constructed in Definition 3.4. Let $\mathcal{G}_{s}$ be an arbitrary member of $\left\{\mathcal{G}_{s} \mid(\overline{\mathcal{G}} \lambda \mathcal{G}) \subseteq \mathcal{G}_{s} \subseteq \overline{\mathcal{G}}\right\}$ :

a) Then, $\mathcal{O}$ is an $O S$-vertex sequence for $\mathcal{G}_{s}$ of size $|\mathcal{O}|=\left|\mathcal{G}_{s}\right|-t-1$.

b) Every bag $\mathcal{Z} \in \mathcal{V}_{\overline{\mathcal{T}}}$ is a positive semidefinite zero forcing set for $\mathcal{G}_{s}$ of size $|\mathcal{Z}|=t+1$.

Proof. Consider the procedure described in Definition 3.4 for the construction of the supergraph $\overline{\mathcal{G}}$. It is easy to verify that $\mathcal{O}$ includes all vertices of $\overline{\mathcal{G}}$ except for those in the only remaining vertex of $\tilde{\mathcal{T}}$ when this process is terminated. Call this bag $V_{1}$. 


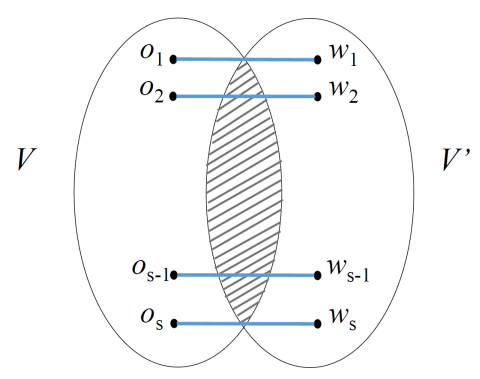

(a)

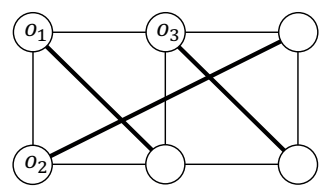

(b)

FIG. 3.1. (a) This figure illustrates Step 3 of Definition 3.4 for designing an enriched supergraph. The shaded area includes the common vertices of bags $V$ and $V^{\prime} ;(b)$ OS-vertex sequence $\mathcal{O}$ for the graph $\mathcal{G}$ depicted in Figure 2.3.
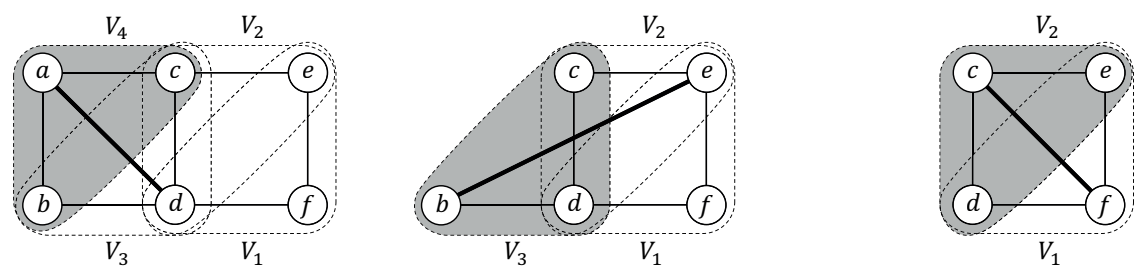

FiG. 3.2. An enriched supergraph $\overline{\mathcal{G}}$ of the graph $\mathcal{G}$ given in Figure 2.3

Hence,

$$
|\mathcal{O}|=|\overline{\mathcal{G}}|-\left|V_{1}\right|=|\overline{\mathcal{G}}|-(t+1) .
$$

Now, it remains to show that $\mathcal{O}$ is an OS-vertex sequence. To this end, let $\mathcal{G}_{s}$ be an arbitrary member of $\left\{\mathcal{G}_{s} \mid(\overline{\mathcal{G}} \lambda \mathcal{G}) \subseteq \mathcal{G}_{s} \subseteq \overline{\mathcal{G}}\right\}$. We use induction to prove that $\mathcal{O}$ is an OS-vertex sequence of $\mathcal{G}_{s}$.

For $|\mathcal{T}|=1$, the sequence $\mathcal{O}$ is empty and the statement is trivial. For $|\mathcal{T}|>1$, consider the first run of the loop in the algorithm. Notice that

$$
\left\{o_{1}, \ldots, o_{s}\right\} \subseteq V \quad \text { and } \quad\left\{o_{1}, \ldots, o_{s}\right\} \cap V^{\prime}=\varnothing .
$$

Let $\overline{\mathcal{T}}_{v}$ denote the subgraph induced by all bags of $\overline{\mathcal{T}}$ that include an arbitrary vertex $v \in \mathcal{G}$. According to the definition of tree decomposition, we have

$$
V \in \overline{\mathcal{T}}_{o} \quad \text { and } \quad V^{\prime} \notin \overline{\mathcal{T}}_{o}
$$

for every $o \in\left\{o_{1}, \ldots, o_{s}\right\}$. Since $\overline{\mathcal{T}}_{o}$ is a connected subgraph of $\overline{\mathcal{T}}$ and $V$ is a leaf, (3.3) implies that $\overline{\mathcal{T}}_{o}$ has one node and no edges, i.e.,

$$
\overline{\mathcal{T}}_{o}=(\{V\}, \varnothing) \text { for } \quad o \in\left\{o_{1}, \ldots, o_{s}\right\}
$$

On the other hand, since $\left\{w_{1}, \ldots, w_{s}\right\} \cap V=\varnothing$, we have

$$
V \notin \mathcal{V}_{\overline{\mathcal{T}}_{w}} \quad \text { for } \quad w \in\left\{w_{1}, \ldots, w_{s}\right\} .
$$

Given a pair $(i, j) \in\{1, \ldots, s\} \times\{1, \ldots, s\}$, the relations (3.4) and 3.5 yield that the trees $\overline{\mathcal{T}}_{o_{i}}$ and $\overline{\mathcal{T}}_{w_{j}}$ do not intersect and therefore $\left(o_{i}, w_{j}\right) \notin \mathcal{E}_{\mathcal{G}}$. Accordingly, since 
the edges $\left(o_{1}, w_{1}\right), \ldots,\left(o_{s}, w_{s}\right)$ are added to the graph at Step 3 of the algorithm, we have

$$
\left(w_{i}, o_{j}\right) \in \mathcal{E}_{\mathcal{G}_{s}} \Longleftrightarrow i=j .
$$

This means that the vertex $o_{i}$ in the sequence $\mathcal{O}$ has a neighbor $w_{i}$ satisfying the requirements of the OS definition (note that $\left(o_{i}, w_{i}\right)$ is an edge of $\mathcal{G}_{s}$ ).

On the other hand, $\overline{\mathcal{T}} \backslash V$ is a tree decomposition for the subgraph of $\overline{\mathcal{G}}$ induced by the vertex subset $\mathcal{V}_{\overline{\mathcal{G}}} \backslash\left\{o_{1}, \ldots, o_{s}\right\}$. Hence, according to the induction assumption, the remaining members of the sequence $\mathcal{O}$ satisfy the conditions of Definition 3.1. This completes the proof of Part (a).

For Part (b), we need to show that by using the procedure explained in Definition 3.3 and starting from an arbitrary bag $\mathcal{Z} \in \mathcal{V}_{\mathcal{T}}$, all of the vertices in $\mathcal{V}_{\overline{\mathcal{G}}}$ can be added to $\mathcal{Z}^{\prime}$ sequentially. To prove this, consider two neighboring bags $\mathcal{Y}_{1}, \mathcal{Y}_{2} \in \mathcal{V}_{\mathcal{T}}$ such that $\mathcal{Y}_{1} \subseteq \mathcal{Z}^{\prime}$ and $\mathcal{Y}_{2} \nsubseteq \mathcal{Z}^{\prime}$. According to the definition of enriched suppergraph, every vertex $y_{2} \in \mathcal{Y}_{2} \backslash \mathcal{Y}_{1}$ is the unique neighbor of only a single vertex $y_{1} \in \mathcal{Y}_{1} \backslash \mathcal{Y}_{2}$ in the set $\mathcal{Y}_{2} \backslash \mathcal{Y}_{1}$ and, therefore, this vertex can be added to $\mathcal{Z}^{\prime}$. Hence, all vertices in $\mathcal{Y}_{2} \backslash \mathcal{Y}_{1}$ can be added to $\mathcal{Z}^{\prime}$ and then we can proceed with neighboring bags until $\overline{\mathcal{G}}$ is covered. $\mathrm{Y}$

COROLlary 3.6. For every graph $\mathcal{G}$, there exists a supergraph $\overline{\mathcal{G}}$ with the property that

$$
\max _{\mathcal{G}_{s}}\left\{\mathrm{Z}^{+}\left(\mathcal{G}_{s}\right) \mid(\overline{\mathcal{G}} \lambda \mathcal{G}) \subseteq \mathcal{G}_{s} \subseteq \overline{\mathcal{G}}\right\} \leq \mathrm{tw}(\mathcal{G})+1
$$

Proof. The proof follows directly from Theorem 3.5 and the equation 1.3 .

4. Low-Rank Solutions Via Graph Decomposition. In this section, we develop a graph-theoretic technique to find a low-rank feasible solution of the LMI problem (1.1). To this end, we first introduce a convex optimization problem.

Optimization A: Let $\mathcal{G}$ and $\mathcal{G}^{\prime}$ be two graphs such that $\mathcal{V}_{\mathcal{G}}=\{1, \ldots, n\}, \mathcal{V}_{\mathcal{G}^{\prime}}=$ $\{1, \ldots, m\}, n \leq m$, and $\mathcal{E}_{\mathcal{G}} \subseteq \mathcal{E}_{\mathcal{G}^{\prime}}$. Consider arbitrary matrices $\mathbf{X}^{\text {ref }} \in \mathbb{F}_{n}^{+}$and $\mathbf{T} \in \mathbb{F}_{m}$ with the property that $\mathscr{G}(\mathbf{T})=\mathcal{G}^{\prime}$, where $\left(\mathbb{F}_{n}^{+}, \mathbb{F}_{m}\right)$ is either $\left(\mathbb{S}_{n}^{+}, \mathbb{S}_{m}\right)$ or $\left(\mathbb{H}_{n}^{+}, \mathbb{H}_{m}\right)$. The problem

$$
\begin{array}{clr}
\underset{\overline{\mathbf{X}} \in \mathbb{F}_{m}}{\operatorname{minimize}} & \operatorname{trace}\{\mathbf{T} \overline{\mathbf{X}}\} & \\
\text { subject to } & \bar{X}_{k k}=X_{k k}^{\text {ref }}, & k \in \mathcal{V}_{\mathcal{G}}, \\
& \bar{X}_{k k}=1 & k \in \mathcal{V}_{\mathcal{G}^{\prime}} \backslash \mathcal{V}_{\mathcal{G}}, \\
& \bar{X}_{i j}=X_{i j}^{\text {ref }} & (i, j) \in \mathcal{E}_{\mathcal{G}}, \\
& \overline{\mathbf{X}} \succeq 0, &
\end{array}
$$

is referred to as "Optimization A with the input $\left(\mathcal{G}, \mathcal{G}^{\prime}, \mathbf{T}, \mathbf{X}^{\text {ref }}\right)$ ".

Optimization A is a convex semidefinite program with a non-empty feasible set containing the point

$$
\left[\begin{array}{c|c}
\mathbf{X}^{\mathrm{ref}} & 0_{n \times(m-n)} \\
\hline 0_{(m-n) \times n} & \mathbf{I}_{(m-n)}
\end{array}\right]
$$

Let $\overline{\mathbf{X}}^{\text {opt }} \in \mathbb{F}_{m}$ denote an arbitrary solution of Optimization A with the input $\left(\mathcal{G}, \mathcal{G}^{\prime}, \mathbf{T}, \mathbf{X}^{\text {ref }}\right)$ and $\mathbf{X}^{\text {opt }} \in \mathbb{F}_{n}$ represent its $n$-th leading principal submatrix. Then, 
$\mathbf{X}^{\text {opt }}$ is called the subsolution to Optimization A associated with $\overline{\mathbf{X}}^{\text {opt }}$. Note that $\mathbf{X}^{\text {opt }}$ and $\mathbf{X}^{\text {ref }}$ share the same diagonal and values for the entries corresponding to the edges of $\mathcal{G}$. Hence, Optimization A is intrinsically a positive semidefinite matrix completion problem with the input $\mathbf{X}^{\text {ref }}$ and the output $\mathbf{X}^{\mathrm{opt}}$. Moreover, it can be easily observed that if $\mathbf{X}^{\text {ref }}$ is feasible for the problem (1.1), then every subsolution of Optimization A is feasible for (1.1) as well.

Definition 4.1 (msr). Given a simple graph $\mathcal{G}$, define the real symmetric and complex Hermitian minimum semidefinite rank of $\mathcal{G}$ as

$$
\begin{aligned}
\operatorname{msr}_{\mathbb{S}}(\mathcal{G}) \triangleq \min \{\operatorname{rank}(\mathbf{W}) \mid \mathscr{G}(\mathbf{W}) & \left.=\mathcal{G}, \mathbf{W} \in \mathbb{S}_{n}^{+}\right\} \\
\operatorname{msr}_{\mathbb{H}}(\mathcal{G}) \triangleq \min \{\operatorname{rank}(\mathbf{W}) \mid \mathscr{G}(\mathbf{W}) & \left.=\mathcal{G}, \mathbf{W} \in \mathbb{H}_{n}^{+}\right\} .
\end{aligned}
$$

The role of Optimization A is to find a low-rank feasible point for the original LMI problem (1.1). This can be achieved by reformulating the LMI problem (1.1) with respect to a possibly higher dimensional matrix $\overline{\mathbf{X}} \in \mathbb{F}^{\left|\mathcal{G}^{\prime}\right|}$, whose rows and columns correspond to the vertices of $\mathcal{G}^{\prime}$. In other words, the low-rank matrix to be sought is embedded in $\overline{\mathbf{X}}$. As illustrated in Examples 1, 2 and 3, we aim to show that if the graph $\mathcal{G}^{\prime}$ in Optimization $\mathrm{A}$ is chosen as an enriched supergraph of $\mathcal{G}$, then the minimization of every nonzero weighted sum of the entries of $\overline{\mathbf{X}}$ corresponding to the edges of $\mathcal{G}^{\prime}$ results in a low-rank solution. In addition to the graph-theoretic analysis required to construct the enriched supergraph, this result requires the availability of an initial positive-definite feasible point $\mathbf{X}^{\text {ref }}$ to built upon. Such an initial matrix $\mathbf{X}^{\text {ref }}$ can be obtained via any interior-point algorithm for the semidefinite program (1.1), after possibly imposing the explicit conic constraint

$$
\mathbf{X} \succeq \varepsilon \times \mathbf{I}_{n}
$$

for a sufficiently small $\varepsilon>0$.

THEOREM 4.2. Assume that $\mathbf{M}_{1}, \ldots, \mathbf{M}_{p}$ are arbitrary matrices in $\mathbb{F}_{n}$ which is equal to either $\mathbb{S}_{n}$ or $\mathbb{H}_{n}$. Suppose that $a_{1}, \ldots, a_{p}$ are real numbers such that the feasibility problem

$$
\begin{array}{ll}
\text { find } & \mathbf{X} \in \mathbb{F}_{n} \\
\text { subject to } & \operatorname{trace}\left\{\mathbf{M}_{k} \mathbf{X}\right\} \leq a_{k}, \quad k=1, \ldots, p, \\
& \mathbf{X} \succeq 0,
\end{array}
$$

has a positive-definite feasible solution $\mathbf{X}^{\mathrm{ref}} \in \mathbb{F}_{n}^{+}$. Let $\mathcal{G}=\mathscr{G}\left(\mathbf{M}_{1}\right) \cup \cdots \cup \mathscr{G}\left(\mathbf{M}_{p}\right)$.

a) Consider an arbitrary supergraph $\mathcal{G}^{\prime}$ of $\mathcal{G}$. Every subsolution $\mathbf{X}^{\mathrm{opt}}$ to Optimization $A$ with the input $\left(\mathcal{G}, \mathcal{G}^{\prime}, \mathbf{T}, \mathbf{X}^{\text {ref }}\right)$ is a solution to the LMI problem 4.5) and satisfies the relations

$$
\begin{aligned}
& \operatorname{rank}\left\{\mathbf{X}^{\mathrm{opt}}\right\} \leq\left|\mathcal{G}^{\prime}\right|-\min _{\mathcal{G}_{s}}\left\{\operatorname{msr}_{\mathbb{F}}\left(\mathcal{G}_{s}\right) \mid\left(\mathcal{G}^{\prime} \lambda \mathcal{G}\right) \subseteq \mathcal{G}_{s} \subseteq \mathcal{G}^{\prime}\right\}, \\
& \operatorname{rank}\left\{\mathbf{X}^{\mathrm{opt}}\right\} \leq \max _{\mathcal{G}_{s}}\left\{\mathrm{Z}^{+}\left(\mathcal{G}_{s}\right) \mid\left(\mathcal{G}^{\prime} \lambda \mathcal{G}\right) \subseteq \mathcal{G}_{s} \subseteq \mathcal{G}^{\prime}\right\} .
\end{aligned}
$$

b) Consider an arbitrary tree decomposition $\mathcal{T}$ of $\mathcal{G}$ with width $t$. Let $\overline{\mathcal{G}}$ be an enriched supergraph of $\mathcal{G}$ derived by $\mathcal{T}$. Every subsolution $\mathbf{X}^{\mathrm{opt}}$ to Optimization $A$ with the input $\left(\mathcal{G}, \overline{\mathcal{G}}, \mathbf{T}, \mathbf{X}^{\mathrm{ref}}\right)$ is a solution to 4.5 and satisfies the relation

$$
\operatorname{rank}\left\{\mathbf{X}^{\mathrm{opt}}\right\} \leq t+1
$$


Proof. To prove Part (a), notice that $X_{i j}$ does not play a role in the linear constraint 4.5a) of the LMI problem 4.5) as long as $i \neq j$ and $(i, j) \notin \mathcal{E}_{\mathcal{G}}$. It can be inferred from this property that $\mathbf{X}^{\mathrm{opt}}$ is a solution of 4.5 . Now, it remains to show the validity of the inequality (4.6). Constraints $4.1 \mathrm{~b}),(4.1 \mathrm{c})$ and $(4.1 \mathrm{~d})$ imply that for every feasible solution $\overline{\mathbf{X}}$ of Optimization A, the matrix $\mathbf{X}-\overline{\mathbf{X}}^{\mathrm{opt}}$ belongs to the convex cone

$$
C=\left\{\mathbf{W} \in \mathbb{F}_{m} \mid W_{k k}=0 \quad \text { for } \quad k \in \mathcal{V}_{\mathcal{G}^{\prime}}, \quad W_{i j}=0 \quad \text { for } \quad(i, j) \in \mathcal{E}_{\mathcal{G}}\right\} .
$$

Therefore, a dual matrix variable $\boldsymbol{\Lambda}$ could be assigned to these constraints, which belongs to the dual cone

$$
C^{\perp}=\left\{\mathbf{W} \in \mathbb{F}_{m} \mid W_{i j}=0 \quad \text { for } \quad(i, j) \notin \mathcal{E}_{\mathcal{G}} \text { and } \mathrm{i} \neq \mathrm{j}\right\} .
$$

Hence, the Lagrangian of Optimization A can be expressed as

$$
\begin{aligned}
\mathcal{L}(\overline{\mathbf{X}}, \boldsymbol{\Lambda}, \boldsymbol{\Phi}) & =\operatorname{trace}\{\mathbf{T} \overline{\mathbf{X}}\}+\operatorname{trace}\left\{\boldsymbol{\Lambda}\left(\overline{\mathbf{X}}-\overline{\mathbf{X}}^{\mathrm{opt}}\right)\right\}-\operatorname{trace}\{\boldsymbol{\Phi} \overline{\mathbf{X}}\} \\
& =\operatorname{trace}\{(\boldsymbol{\Lambda}+\mathbf{T}-\mathbf{\Phi}) \overline{\mathbf{X}}\}-\operatorname{trace}\left\{\boldsymbol{\Lambda} \overline{\mathbf{X}}^{\mathrm{opt}}\right\}
\end{aligned}
$$

where $\mathbf{\Phi} \succeq 0$ denotes the matrix dual variable corresponding to the constraint $\overline{\mathbf{X}} \succeq 0$. The infimum of the Lagrangian over $\overline{\mathbf{X}}$ is $-\infty$ unless $\boldsymbol{\Phi}=\boldsymbol{\Lambda}+\mathbf{T}$. Therefore, the dual problem is as follows:

$$
\begin{array}{ll}
\underset{\boldsymbol{\Lambda} \in \mathbb{F}_{m}}{\operatorname{maximize}} & -\operatorname{trace}\left\{\boldsymbol{\Lambda} \overline{\mathbf{X}}^{\text {opt }}\right\} \\
\text { subject to } & \Lambda_{i j}=0
\end{array} \quad(i, j) \notin \mathcal{E}_{\mathcal{G}} \text { and } \mathrm{i} \neq \mathrm{j},
$$

By pushing the diagonal entries of $\boldsymbol{\Lambda}$ toward infinity, the inequality $\boldsymbol{\Lambda}+\mathbf{T} \succeq 0$ will become strict. Hence, strong duality holds according to the Slater's condition. If $\boldsymbol{\Phi}=\boldsymbol{\Phi}^{\text {opt }}$ denotes an arbitrary dual solution, the complementary slackness condition $\operatorname{trace}\left\{\boldsymbol{\Phi}^{\mathrm{opt}} \overline{\mathbf{X}}^{\mathrm{opt}}\right\}=0$ yields that

$$
\operatorname{rank}\left\{\boldsymbol{\Phi}^{\mathrm{opt}}\right\}+\operatorname{rank}\left\{\overline{\mathbf{X}}^{\mathrm{opt}}\right\} \leq m
$$

(note that since the primal and dual problems are strictly feasible, $\overline{\mathbf{X}}^{\text {opt }}$ and $\boldsymbol{\Phi}^{\text {opt }}$ are both finite). On the other hand, according to the equations $\boldsymbol{\Phi}=\boldsymbol{\Lambda}+\mathbf{T}$ and $\boldsymbol{\Lambda} \in C^{\perp}$, we have

$$
\begin{array}{ll}
\boldsymbol{\Phi}_{i j}^{\text {opt }} \neq 0, & \text { for } \quad(\mathrm{i}, \mathrm{j}) \in \mathcal{E}_{\mathcal{G}^{\prime}} \backslash \mathcal{E}_{\mathcal{G}} \\
\boldsymbol{\Phi}_{i j}^{\text {opt }}=0, & \text { for } \quad(\mathrm{i}, \mathrm{j}) \notin \mathcal{E}_{\mathcal{G}^{\prime}} \text { and } \mathrm{i} \neq \mathrm{j} .
\end{array}
$$

Therefore,

$$
\left(\mathcal{G}^{\prime} \lambda \mathcal{G}\right) \subseteq \mathscr{G}\left(\mathbf{\Phi}^{\mathrm{opt}}\right) \subseteq \mathcal{G}^{\prime}
$$

The first upper bound on $\operatorname{rank}\left\{\mathbf{X}^{\text {opt }}\right\}$ given in 4.6a can be obtained by combining 4.12 and 4.14 after noting that $\operatorname{rank}\left\{\mathbf{X}^{\text {opt }}\right\} \leq \operatorname{rank}\left\{\overline{\mathbf{X}}^{\text {opt }}\right\}$ (recall that $\mathbf{X}^{\text {opt }}$ is a submatrix of $\overline{\mathbf{X}}^{\mathrm{opt}}$ ). In addition, according to the equation $(1.3)$, we have:

$$
\begin{aligned}
\operatorname{rank}\left\{\mathbf{X}^{\mathrm{opt}}\right\} & \leq|\overline{\mathcal{G}}|-\min \left\{\operatorname{msr}_{\mathbb{F}}\left(\mathcal{G}_{s}\right) \mid(\overline{\mathcal{G}} \lambda \mathcal{G}) \subseteq \mathcal{G}_{s} \subseteq \overline{\mathcal{G}}\right\} \\
& \leq|\overline{\mathcal{G}}|-\min \left\{\operatorname{OS}\left(\mathcal{G}_{s}\right) \mid(\overline{\mathcal{G}} \lambda \mathcal{G}) \subseteq \mathcal{G}_{s} \subseteq \overline{\mathcal{G}}\right\} \\
& \leq \max \left\{\mathrm{Z}^{+}\left(\mathcal{G}_{s}\right) \mid(\overline{\mathcal{G}} \lambda \mathcal{G}) \subseteq \mathcal{G}_{s} \subseteq \overline{\mathcal{G}}\right\}
\end{aligned}
$$


This completes the proof of Part (a).

For Part (b), it follows from Theorem 3.5 that $\operatorname{OS}\left(\mathcal{G}_{s}\right) \geq|\overline{\mathcal{G}}|-t-1$ for every $\mathcal{G}_{s}$ with the property $(\overline{\mathcal{G}} \backslash \mathcal{G}) \subseteq \mathcal{G}_{s} \subseteq \overline{\mathcal{G}}$. Therefore,

$$
\begin{aligned}
\operatorname{rank}\left\{\mathbf{X}^{\mathrm{opt}}\right\} & \leq|\overline{\mathcal{G}}|-\min \left\{\operatorname{msr}_{\mathbb{F}}\left(\mathcal{G}_{s}\right) \mid(\overline{\mathcal{G}} 入 \mathcal{G}) \subseteq \mathcal{G}_{s} \subseteq \overline{\mathcal{G}}\right\} \\
& \leq|\overline{\mathcal{G}}|-\min \left\{\operatorname{OS}\left(\mathcal{G}_{s}\right) \mid(\overline{\mathcal{G}} \lambda \mathcal{G}) \subseteq \mathcal{G}_{s} \subseteq \overline{\mathcal{G}}\right\} \\
& \leq|\overline{\mathcal{G}}|-(|\overline{\mathcal{G}}|-t-1) \\
& \leq t+1
\end{aligned}
$$

(note that $\operatorname{OS}(\mathcal{G}) \leq \operatorname{msr}_{\mathbb{F}}(\mathcal{G})$ as proven in $[19$ ). This completes the proof.

Observe that the objective function of Optimization A is a weighted sum of certain entries of the matrix $\overline{\mathbf{X}}$, where the weights come from the matrix $\mathbf{T}$. Part (a) of Theorem 4.2 proposes an upper bound on the rank of all subsolutions of this optimization, which is contingent upon the graph of the weight matrix $\mathbf{T}$ without making use of the nonzero values of the weights.

COROLLARY 4.3. If the LMI problem 4.5) has a positive-definite feasible solution, then it has a solution $\mathbf{X}^{\mathrm{opt}}$ with rank at most $\mathrm{tw}(\mathcal{G})+1$.

Proof. The proof follows immediately from Part (b) of Theorem 4.2 by considering $\mathcal{T}$ to be a minimal tree decomposition of $\mathcal{G}$. $\square$

Note that Theorem 4.2 and Corollary 4.3 both require the existence of a positivedefinite feasible solution. This assumption will be reduced to only the feasibility of the LMI problem 4.5 in the next section.

We now revisit Examples 1, 2 and 3 provided earlier and study them using Theorem 4.2. First, consider Example 1. The graph $\mathcal{G}$ corresponding to a matrix $\mathbf{X}$ with known diagonal entries has the vertex set $\{1,2, \ldots, n\}$ with no edges. An enriched supergraph graph $\overline{\mathcal{G}}$ can be obtained from $\mathcal{G}$ by connecting vertices $i$ and $i+1$ for $i=1, \ldots, n-1$. Consider an arbitrary matrix $\mathbf{T} \in \mathbf{S}^{n}$ with the representative graph $\overline{\mathcal{G}}$. This matrix is sparse with nonzero subdiagonal and superdiagonal. Using Theorem 4.2 Optimization A yields a solution such that $\mathbf{X}^{\mathrm{opt}} \leq \operatorname{tw}(\mathcal{G})+1$. Since $\mathcal{G}$ does not have any edges, its treewidth is equal to 0 . As a result, every solution of Optimization A has rank 1.

Consider now Example 2 with $\mathbf{X}$ visualized in Figure 1.1. As can be observed, two $2 \times 2$ blocks of $\mathbf{X}$ specified by dashed red lines are known and the goal is to design the block $\mathbf{X}_{31}$. The graph $\mathcal{G}$ has $n=\alpha+\beta+\gamma$ vertices with the property that the subgraphs induced by the vertex subsets $\{1, \ldots, \alpha+\beta\}$ and $\{\alpha+1, \ldots, n\}$ are both complete graphs. In the case where $\alpha=\gamma$, an enriched supergraph $\overline{\mathcal{G}}$ can be obtained by connecting vertex $i$ to vertex $\alpha+\beta+i$ for $i=1,2, \ldots, \alpha$. Consider a matrix $\mathbf{T}$ with the representative graph $\overline{\mathcal{G}}$. Optimization A then aims to minimize the weighted sum over the diagonal entries of $\mathbf{X}_{31}$. Consider now the case where $\alpha>\gamma$. A tree decomposition of $\mathcal{G}$ has two bags $\{1, \ldots, \alpha+\beta\}$ and $\{\alpha+1, \ldots, \alpha+\beta+\gamma\}$. Since these bags have disparate sizes, the definition of enriched supergraph requires adding $\alpha-\gamma$ new vertices to the bag with the fewer number of vertices. This can be translated as adding $\alpha-\gamma$ rows and $\alpha-\gamma$ columns to $\mathbf{X}$ in order to arrive at the augmented matrix $\widehat{X}$ depicted in Figure 1.1(b). In this case, Optimization A may minimize a weighted sum of the diagonal entries of the square block including $\widehat{\mathbf{X}}_{31}$ and $\widehat{\mathbf{X}}_{41}$. Regarding Example 3, the matrix $\mathcal{G}$ has the vertex set $\mathcal{V}_{\mathcal{G}}=\{1, \ldots, 4 \alpha\}$ such that its subgraphs induced by the vertex subsets $\{1, \ldots, 2 \alpha\},\{\alpha+1, \ldots, 3 \alpha\}$, and $\{2 \alpha+1, \ldots, 4 \alpha\}$ are all complete graphs. A tree decomposition of $\mathcal{G}$ has three bags $\{1, \ldots, 2 \alpha\},\{\alpha+1, \ldots, 3 \alpha\}$ and $\{2 \alpha+1, \ldots, 4 \alpha\}$. Hence, an enriched graph $\overline{\mathcal{G}}$ 
can be obtained by connecting vertices $i$ and $2 \alpha+i$ as well as vertices $i+\alpha$ and $3 \alpha+i$ for $i=1, \ldots, \alpha$. This implies that Optimization A minimizes a weighted sum of the diagonal entries of the blocks $\mathbf{X}_{31}$ and $\mathbf{X}_{42}$.

In the next section, we have applied a combined graph-theoretic and algebraic approach in order to strengthened the results of Theorem 4.2 .

5. Combined Graph-Theoretic and Algebraic Method. In this section, a combined graph-theoretic and algebraic method is developed in order to strengthen the results of the preceding section. According to Theorem 4.2 the minimization of a weighted sum of certain unknown entries of a partially-known matrix (through Optimization A) results in a low-rank positive semidefinite completion, where the entries to be minimized can be found via a graph-theoretic analysis. The above result requires the existence of a positive-definite feasible solution for the LMI problem 4.5. The first objective of this section is to relax this assumption to the existence of only a feasible solution. The second objective is to further characterize the rank of the resulting solution as an extension to Theorem 4.2 and Corollary 4.3

Given an arbitrary matrix $\mathbf{M}$ in $\mathbb{F}_{n}$, we denote its Moore-Penrose pseudoinverse as $\mathbf{M}^{+}$. If $r=\operatorname{rank}\{\mathbf{M}\}$ and $\mathbf{M}$ admits the eigenvalue decomposition $\mathbf{M}=$ $\mathbf{Q} \boldsymbol{\Lambda} \mathbf{Q}^{*}$ with $\boldsymbol{\Lambda}=\operatorname{diag}\left\{\left[\lambda_{1}, \ldots, \lambda_{r}, 0, \ldots, 0\right]\right\}$, then $\mathbf{M}^{+}=\mathbf{Q} \boldsymbol{\Lambda}^{+} \mathbf{Q}^{*}$ where $\boldsymbol{\Lambda}^{+}=$ $\operatorname{diag}\left\{\left[\lambda_{1}^{-1}, \ldots, \lambda_{r}^{-1}, 0, \ldots, 0\right]\right\}$. The next lemma is borrowed from [50].

LEMma 5.1. Given a $2 \times 2$ block matrix

$$
\mathbf{M}=\left[\begin{array}{cc}
\mathbf{A} & \mathbf{B}^{*} \\
\mathbf{B} & \mathbf{C}
\end{array}\right] \in \mathbb{F}_{n},
$$

define its generalized Schur complement as $\mathbf{S}^{+} \triangleq \mathbf{C}-\mathbf{B A}^{+} \mathbf{B}^{*}$. The relation $\mathbf{M} \succeq 0$ holds if and only if

$$
\mathbf{A} \succeq 0, \quad \mathbf{S}^{+} \succeq 0 \quad \text { and } \quad \operatorname{null}\{\mathbf{A}\} \subseteq \operatorname{null}\{\mathbf{B}\} .
$$

In addition, the equation $\operatorname{rank}\{\mathbf{M}\}=\operatorname{rank}\{\mathbf{A}\}+\operatorname{rank}\left\{\mathbf{S}^{+}\right\}$is satisfied if and only if $\operatorname{null}\{\mathbf{A}\} \subseteq \operatorname{null}\{\mathbf{B}\}$.

THEOREM 5.2. Consider the block matrix

$$
\mathbf{M}(\mathbf{U}) \triangleq\left[\begin{array}{ccc}
\mathbf{A} & \mathbf{B}_{x}^{*} & \mathbf{B}_{y}^{*} \\
\mathbf{B}_{x} & \mathbf{X} & \mathbf{U}^{*} \\
\mathbf{B}_{y} & \mathbf{U} & \mathbf{Y}
\end{array}\right]
$$

where $\mathbf{A}, \mathbf{X}, \mathbf{Y}, \mathbf{B}_{x}^{*}$ and $\mathbf{B}_{y}^{*}$ are known and the matrix $\mathbf{U}$ is the variable. Define

$$
\mathbf{M}_{x} \triangleq\left[\begin{array}{cc}
\mathbf{A} & \mathbf{B}_{x}^{*} \\
\mathbf{B}_{x} & \mathbf{X}
\end{array}\right] \text { and } \mathbf{M}_{y} \triangleq\left[\begin{array}{cc}
\mathbf{A} & \mathbf{B}_{y}^{*} \\
\mathbf{B}_{y} & \mathbf{Y}
\end{array}\right] .
$$

Define also $\mathbf{S}_{x}^{+} \triangleq \mathbf{X}-\mathbf{B}_{x} \mathbf{A}^{+} \mathbf{B}_{x}^{*}$ and $\mathbf{S}_{y}^{+} \triangleq \mathbf{Y}-\mathbf{B}_{y} \mathbf{A}^{+} \mathbf{B}_{y}^{*}$. Given a constant matrix $\mathbf{T}$ of appropriate dimension, for every solution $\mathbf{U}^{\text {opt }}$ of the optimization problem

$$
\begin{array}{ll}
\underset{\mathbf{U}}{\operatorname{minimize}} & \operatorname{trace}\left\{\mathbf{T} \mathbf{U}^{*}\right\} \\
\text { subject to } & \mathbf{M}(\mathbf{U}) \succeq 0,
\end{array}
$$

$\mathbf{M}\left(\mathbf{U}^{\text {opt }}\right)$ has the minimum possible rank, i.e.,

$$
\operatorname{rank}\left\{\mathbf{M}\left(\mathbf{U}^{\mathrm{opt}}\right)\right\}=\max \left\{\operatorname{rank}\left\{\mathbf{M}_{x}\right\}, \operatorname{rank}\left\{\mathbf{M}_{y}\right\}\right\}
$$


provided that $\mathbf{S}_{y}^{+} \mathbf{T} \mathbf{S}_{x}^{+}$has the maximum possible rank, i.e.,

$$
\operatorname{rank}\left\{\mathbf{S}_{y}^{+} \mathbf{T} \mathbf{S}_{x}^{+}\right\}=\min \left\{\operatorname{rank}\left\{\mathbf{S}_{x}^{+}\right\}, \operatorname{rank}\left\{\mathbf{S}_{y}^{+}\right\}\right\} .
$$

Proof. Let $r_{x} \triangleq \operatorname{rank}\left\{\mathbf{S}_{x}^{+}\right\}$and $r_{y} \triangleq \operatorname{rank}\left\{\mathbf{S}_{y}^{+}\right\}$. Consider the following eigenvalue decompositions for $\mathbf{S}_{x}^{+}$and $\mathbf{S}_{y}^{+}$:

$$
\mathbf{S}_{x}^{+}=\mathbf{Q}_{x} \boldsymbol{\Lambda}_{x} \mathbf{Q}_{x}^{*} \quad \text { and } \quad \mathbf{S}_{y}^{+}=\mathbf{Q}_{y} \boldsymbol{\Lambda}_{y} \mathbf{Q}_{y}^{*} .
$$

Let $\mathbf{Q}_{x}=\left[\begin{array}{ll}\mathbf{Q}_{x 1} & \mathbf{Q}_{x 0}\end{array}\right]$ and $\mathbf{Q}_{y}=\left[\begin{array}{ll}\mathbf{Q}_{y 1} & \mathbf{Q}_{y 0}\end{array}\right]$, where $\mathbf{Q}_{x 1} \in \mathbb{F}^{n \times r_{x}}$ and $\mathbf{Q}_{y 1} \in \mathbb{F}^{n \times r_{y}}$. We can also write

$$
\boldsymbol{\Lambda}_{x} \triangleq\left[\begin{array}{cc}
\boldsymbol{\Lambda}_{x 1} & \mathbf{0} \\
\mathbf{0} & \mathbf{0}
\end{array}\right] \text { and } \boldsymbol{\Lambda}_{y} \triangleq\left[\begin{array}{cc}
\boldsymbol{\Lambda}_{y 1} & \mathbf{0} \\
\mathbf{0} & \mathbf{0}
\end{array}\right],
$$

where $\boldsymbol{\Lambda}_{x 1}$ and $\boldsymbol{\Lambda}_{y 1}$ are diagonal matrices in $\mathbb{F}_{n_{x}}$ and $\mathbb{F}_{n_{y}}$, respectively. Define

$$
\mathbf{E}_{i j} \triangleq \mathbf{Q}_{y i}^{*}\left(\mathbf{U}-\mathbf{B}_{y} \mathbf{A}^{+} \mathbf{B}_{x}^{*}\right) \mathbf{Q}_{x j} \quad \text { for } \quad i, j \in\{1,2\} .
$$

It can be shown that

$$
\begin{aligned}
\mathbf{U}-\mathbf{B}_{y} \mathbf{A}^{+} \mathbf{B}_{x}^{*} & =\left[\begin{array}{ll}
\mathbf{Q}_{y 1} & \mathbf{Q}_{y 0}
\end{array}\right]\left[\begin{array}{ll}
\mathbf{E}_{11} & \mathbf{E}_{10} \\
\mathbf{E}_{01} & \mathbf{E}_{00}
\end{array}\right]\left[\begin{array}{l}
\mathbf{Q}_{x 1}^{*} \\
\mathbf{Q}_{x 0}^{*}
\end{array}\right] \\
& =\mathbf{Q}_{y 1} \mathbf{E}_{11} \mathbf{Q}_{x 1}^{*}+\mathbf{Q}_{y 1} \mathbf{E}_{10} \mathbf{Q}_{x 0}^{*}+\mathbf{Q}_{y 0} \mathbf{E}_{01} \mathbf{Q}_{x 1}^{*}+\mathbf{Q}_{y 0} \mathbf{E}_{00} \mathbf{Q}_{x 0}^{*} .
\end{aligned}
$$

Hence,

$$
\begin{aligned}
\mathbf{S}^{+} & \triangleq\left[\begin{array}{cc}
\mathbf{X} & \mathbf{U}^{*} \\
\mathbf{U} & \mathbf{Y}
\end{array}\right]-\left[\begin{array}{c}
\mathbf{B}_{x} \\
\mathbf{B}_{y}
\end{array}\right] \mathbf{A}^{+}\left[\begin{array}{ll}
\mathbf{B}_{x}^{*} & \mathbf{B}_{y}^{*}
\end{array}\right] \\
& =\left[\begin{array}{cc}
\mathbf{Q}_{x 1} \mathbf{\Lambda}_{x 1} \mathbf{Q}_{x 1}^{*} & \mathbf{U}^{*}-\mathbf{B}_{y} \mathbf{A}^{+} \mathbf{B}_{x}^{*} \\
\mathbf{U}-\mathbf{B}_{y} \mathbf{A}^{+} \mathbf{B}_{x}^{*} & \mathbf{Q}_{y 1} \mathbf{\Lambda}_{y 1} \mathbf{Q}_{y 1}^{*}
\end{array}\right] .
\end{aligned}
$$

The constraint $\mathbf{M}(\mathbf{U}) \succeq 0$ yields $\mathbf{S}^{+} \succeq 0$ and therefore

$$
\begin{aligned}
{\left[\begin{array}{cc}
\mathbf{0} & \mathbf{E}_{i j}^{*} \\
\mathbf{E}_{i j} & \mathbf{0}
\end{array}\right] } & =\left[\begin{array}{cc}
\mathbf{Q}_{x i}^{*} & \mathbf{0} \\
\mathbf{0} & \mathbf{Q}_{y j}^{*}
\end{array}\right]\left[\begin{array}{cc}
\mathbf{Q}_{x 1} \mathbf{\Lambda}_{x 1} \mathbf{Q}_{x 1}^{*} & \mathbf{U}^{*}-\mathbf{B}_{y} \mathbf{A}^{+} \mathbf{B}_{x}^{*} \\
\mathbf{U}-\mathbf{B}_{y} \mathbf{A}^{+} \mathbf{B}_{x}^{*} & \mathbf{Q}_{y 1} \mathbf{\Lambda}_{y 1} \mathbf{Q}_{y 1}^{*}
\end{array}\right]\left[\begin{array}{cc}
\mathbf{Q}_{x i} & \mathbf{0} \\
\mathbf{0} & \mathbf{Q}_{y j}
\end{array}\right] \\
& =\left[\begin{array}{cc}
\mathbf{Q}_{x i}^{*} & \mathbf{0} \\
\mathbf{0} & \mathbf{Q}_{y j}^{*}
\end{array}\right] \mathbf{S}^{+}\left[\begin{array}{cc}
\mathbf{Q}_{x i} & \mathbf{0} \\
\mathbf{0} & \mathbf{Q}_{y j}
\end{array}\right] \succeq 0 \Longrightarrow \mathbf{E}_{i j}=0,
\end{aligned}
$$

for every $(i, j) \in\{(0,0),(1,0),(0,1)\}$. As a result, the block $\mathbf{U}$ can be written as $\mathbf{U}=\mathbf{B}_{y} \mathbf{A}^{+} \mathbf{B}_{x}^{*}+\mathbf{Q}_{y 1} \mathbf{U}_{1} \mathbf{Q}_{x 1}^{*}$, where $\mathbf{U}_{1} \triangleq \mathbf{E}_{11} \in \mathbb{F}^{r_{y} \times r_{x}}$. Therefore,

$$
\begin{aligned}
\mathbf{S}^{+} & \triangleq\left[\begin{array}{cc}
\mathbf{X} & \mathbf{U}^{*} \\
\mathbf{U} & \mathbf{Y}
\end{array}\right]-\left[\begin{array}{c}
\mathbf{B}_{x} \\
\mathbf{B}_{y}
\end{array}\right] \mathbf{A}^{+}\left[\begin{array}{ll}
\mathbf{B}_{x}^{*} & \mathbf{B}_{y}^{*}
\end{array}\right] \\
& =\left[\begin{array}{cc}
\mathbf{Q}_{x 1} & 0 \\
0 & \mathbf{Q}_{y 1}
\end{array}\right]\left[\begin{array}{cc}
\boldsymbol{\Lambda}_{x 1} & \mathbf{U}_{1}^{*} \\
\mathbf{U}_{1} & \mathbf{\Lambda}_{y 1}
\end{array}\right]\left[\begin{array}{cc}
\mathbf{Q}_{x 1}^{*} & 0 \\
0 & \mathbf{Q}_{y 1}^{*}
\end{array}\right] .
\end{aligned}
$$

Since $\mathbf{M}_{x}, \mathbf{M}_{y} \succeq 0$ according to Lemma 5.1 . one can write

$$
\operatorname{null}\{\mathbf{A}\} \subseteq \operatorname{null}\left\{\mathbf{B}_{x}\right\} \quad \text { and } \operatorname{null}\{\mathbf{A}\} \subseteq \operatorname{null}\left\{\mathbf{B}_{y}\right\}
$$


and therefore Lemma 5.1 yields

$$
\begin{aligned}
\operatorname{rank}\left\{\mathbf{M}_{x}\right\} & =\operatorname{rank}\{\mathbf{A}\}+r_{x} \\
\operatorname{rank}\left\{\mathbf{M}_{y}\right\} & =\operatorname{rank}\{\mathbf{A}\}+r_{y} .
\end{aligned}
$$

This implies that

$$
\mathbf{M} \succeq 0 \quad \Longrightarrow \quad\left[\begin{array}{cc}
\boldsymbol{\Lambda}_{x 1} & \mathbf{U}_{1}^{*} \\
\mathbf{U}_{1} & \boldsymbol{\Lambda}_{y 1}
\end{array}\right] \succeq 0
$$

On the other hand,

$$
\begin{aligned}
\operatorname{trace}\left\{\mathbf{T} \mathbf{U}^{*}\right\} & =\operatorname{trace}\left\{\mathbf{T}\left(\mathbf{B}_{y} \mathbf{A}^{+} \mathbf{B}_{x}^{*}+\mathbf{Q}_{y 1} \mathbf{U}_{1} \mathbf{Q}_{x 1}^{*}\right)^{*}\right\} \\
& =\operatorname{trace}\left\{\mathbf{B}_{y}^{*} \mathbf{T} \mathbf{B}_{x} \mathbf{A}^{+}\right\}+\operatorname{trace}\left\{\mathbf{Q}_{y 1}^{*} \mathbf{T} \mathbf{Q}_{x 1} \mathbf{U}_{1}^{*}\right\} .
\end{aligned}
$$

Hence, the problem 5.5 is equivalent to

$$
\begin{array}{ll}
\underset{\mathbf{U}_{1}}{\operatorname{minimize}} & \operatorname{trace}\left\{\mathbf{T}_{1} \mathbf{U}_{1}^{*}\right\} \\
\text { subject to } & {\left[\begin{array}{cc}
\boldsymbol{\Lambda}_{x 1} & \mathbf{U}_{1}^{*} \\
\mathbf{U}_{1} & \boldsymbol{\Lambda}_{y 1}
\end{array}\right] \succeq 0 .}
\end{array}
$$

where $\mathbf{T}_{1}=\mathbf{Q}_{y 1}^{*} \mathbf{T} \mathbf{Q}_{x 1}$. Let $\mathbf{U}_{1}^{\text {opt }}$ be an arbitrary solution of the above problem. It can be easily seen that the dual matrix variable corresponding to the sole constraint of this problem is equal to

$$
\left[\begin{array}{cc}
\boldsymbol{\Gamma}_{x 1} & \mathbf{T}_{1}^{*} \\
\mathbf{T}_{1} & \boldsymbol{\Gamma}_{y 1}
\end{array}\right] \succeq 0
$$

for some matrices $\boldsymbol{\Gamma}_{x 1}$ and $\boldsymbol{\Gamma}_{y 1}$ (note that both primal and dual solutions are attainable, since the feasible set of the primal problem is bounded and $\mathbf{U}_{1}=0$ serves as a strictly feasible solution). It follows from the complementary slackness that

$$
\operatorname{trace}\left\{\left[\begin{array}{cc}
\boldsymbol{\Lambda}_{x 1} & \mathbf{U}_{1}^{*} \\
\mathbf{U}_{1} & \boldsymbol{\Lambda}_{y 1}
\end{array}\right]\left[\begin{array}{cc}
\boldsymbol{\Gamma}_{x 1} & \mathbf{T}_{1}^{*} \\
\mathbf{T}_{1} & \boldsymbol{\Gamma}_{y 1}
\end{array}\right]\right\}=0
$$

implying that

$$
\operatorname{rank}\left\{\left[\begin{array}{cc}
\boldsymbol{\Lambda}_{x 1} & \mathbf{U}_{1}^{*} \\
\mathbf{U}_{1} & \boldsymbol{\Lambda}_{y 1}
\end{array}\right]\right\}+\operatorname{rank}\left\{\left[\begin{array}{cc}
\boldsymbol{\Gamma}_{x 1} & \mathbf{T}_{1}^{*} \\
\mathbf{T}_{1} & \boldsymbol{\Gamma}_{y 1}
\end{array}\right]\right\} \leq r_{x}+r_{y}
$$

Therefore,

$$
\operatorname{rank}\left\{\left[\begin{array}{cc}
\boldsymbol{\Lambda}_{x 1} & \left(\mathbf{U}_{1}^{\mathrm{opt}}\right)^{*} \\
\mathbf{U}_{1}^{\mathrm{opt}} & \boldsymbol{\Lambda}_{y 1}
\end{array}\right]\right\} \leq r_{x}+r_{y}-\operatorname{rank}\left\{\mathbf{T}_{1}\right\}=r_{x}+r_{y}-\operatorname{rank}\left\{\mathbf{S}_{y}^{+} \mathbf{T} \mathbf{S}_{x}^{+}\right\}
$$

Moreover, it can be concluded from t5.15 that

$$
\operatorname{null}\{\mathbf{A}\} \subseteq \operatorname{null}\left\{\mathbf{B}_{x}\right\} \cap \operatorname{null}\left\{\mathbf{B}_{y}\right\}=\operatorname{null}\left\{\left[\begin{array}{l}
\mathbf{B}_{x} \\
\mathbf{B}_{y}
\end{array}\right]\right\} .
$$

The proof is now completed by Lemma 5.1.

Note that the condition (5.7) required in Theorem 5.2 is satisfied for a generic matrix $\mathbf{T}$. 
Suppose that $\mathbf{O} \in \mathbb{F}^{r_{y} \times r_{x}}$ is a matrix with 1's on its rectangular diagonal and 0 elsewhere. If the matrix $\mathbf{M}(\mathbf{U})$ is completed as

$$
\mathbf{U}=\mathbf{B}_{y} \mathbf{A}^{+} \mathbf{B}_{x}^{*}+\mathbf{Q}_{y 1} \sqrt{\Lambda_{y 1}} \mathbf{O} \sqrt{\Lambda_{x 1}} \mathbf{Q}_{x 1}^{*}
$$

then, it satisfies the rank property (5.6). This explicit formula provides an iterative matrix-completion method.

Definition 5.3. For every matrix $\mathbf{X} \in \mathbb{F}_{k}$ and sets $A, B \subseteq\{1, \ldots, k\}$, define $\mathbf{X}(A, B)$ as a submatrix of $\mathbf{X}$ obtained by choosing those rows of $\mathbf{X}$ with indices appearing in $A$ and those columns of $\mathbf{X}$ with indices in $B$. If $A=B$, then $\mathbf{X}(A, B)$ will be abbreviated as $\mathbf{X}(A)$.

Assume that $\mathbf{M}_{1}, \ldots, \mathbf{M}_{p}$ are arbitrary matrices in $\mathbb{F}_{n}$, which is equal to either $\mathbb{S}_{n}$ or $\mathbb{H}_{n}$. Suppose that $a_{1}, \ldots, a_{p}$ are real numbers such that the feasibility problem

$$
\begin{array}{ll}
\text { find } & \mathbf{X} \in \mathbb{F}_{n} \\
\text { subject to } & \operatorname{trace}\left\{\mathbf{M}_{k} \mathbf{X}\right\} \leq a_{k}, \quad k=1, \ldots, p, \\
& \mathbf{X} \succeq 0,
\end{array}
$$

has a feasible solution $\mathbf{X}^{\text {ref }} \in \mathbb{F}_{n}$. Let $\mathcal{G}=\mathscr{G}\left(\mathbf{M}_{1}\right) \cup \cdots \cup \mathscr{G}\left(\mathbf{M}_{p}\right)$. Consider an arbitrary tree decomposition $\mathcal{T}$ of $\mathcal{G}$ with the set of bags $\mathcal{V}_{\mathcal{T}}=\left\{V_{1}, \ldots, V_{|\mathcal{T}|}\right\}$. Let

$$
r \triangleq \max \left\{\operatorname{rank}\left\{\mathbf{X}^{\mathrm{ref}}\left(V_{k}\right)\right\}|1 \leq k \leq| \mathcal{T} \mid\right\}
$$

and define $\overline{\mathcal{G}}$ as a graph obtained from $\mathcal{G}$ by adding

$$
\sum_{k=1}^{|\mathcal{T}|}\left(r-\operatorname{rank}\left\{\mathbf{X}^{\mathrm{ref}}\left(V_{k}\right)\right\}\right)
$$

new isolated vertices. Let $\overline{\mathcal{T}} \triangleq\left(\overline{\mathcal{V}}_{\mathcal{T}}, \mathcal{E}_{\mathcal{T}}\right)$ be a tree decomposition for $\overline{\mathcal{G}}$ with the bags $\bar{V}_{1}, \ldots, \bar{V}_{|\mathcal{T}|}$, where each bag $\bar{V}_{k}$ is constructed from $V_{k}$ by adding $r-\operatorname{rank}\left\{\mathbf{X}^{\operatorname{ref}}\left(V_{k}\right)\right\}$ of the new isolated vertices in $\mathcal{V}_{\overline{\mathcal{G}}} \backslash \mathcal{V}_{\mathcal{G}}$ such that $\left(\bar{V}_{i} \backslash V_{i}\right) \cap\left(\bar{V}_{j} \backslash V_{j}\right)=\emptyset$ for every $i \neq j$. Let $m \triangleq|\overline{\mathcal{G}}|$ and define the matrix $\overline{\mathbf{X}}^{\text {ref }} \in \mathbb{F}_{m}$ as

$$
\begin{aligned}
& \bar{X}_{k k}^{\mathrm{ref}}=X_{k k}^{\mathrm{ref}} \quad \text { for } \quad k \in \mathcal{V}_{\mathcal{G}} \\
& \bar{X}_{k k}^{\mathrm{ref}}=1 \quad \text { for } \quad k \in \mathcal{V}_{\overline{\mathcal{G}}} \backslash \mathcal{V}_{\mathcal{G}} \\
& \bar{X}_{i j}^{\mathrm{ref}}=X_{i j}^{\mathrm{ref}} \quad \text { for } \quad(i, j) \in \mathcal{E}_{\mathcal{G}} \\
& \bar{X}_{i j}^{\mathrm{ref}}=0 \quad \text { for } \quad(i, j) \notin \mathcal{E}_{\mathcal{G}} .
\end{aligned}
$$

For every pair $i, j \in\{1, \ldots,|\mathcal{T}|\}$, define

$$
\begin{aligned}
\mathbf{S}_{i j}^{+} & \triangleq \overline{\mathbf{X}}^{\mathrm{ref}}\left(\bar{V}_{i} \backslash \bar{V}_{j}\right) \\
& -\overline{\mathbf{X}}^{\mathrm{ref}}\left(\bar{V}_{i} \backslash \bar{V}_{j}, \bar{V}_{i} \cap \bar{V}_{j}\right)\left(\overline{\mathbf{X}}^{\mathrm{ref}}\left(\bar{V}_{i} \cap \bar{V}_{j}\right)\right)^{+} \overline{\mathbf{X}}^{\mathrm{ref}}\left(\bar{V}_{i} \cap \bar{V}_{j}, \bar{V}_{i} \backslash \bar{V}_{j}\right) .
\end{aligned}
$$

Let the edges of the tree decomposition $\mathcal{T}$ be oriented in such a way that the indegree of every node becomes less than or equal to 1 . The resulting directed tree is denoted as $\overrightarrow{\mathcal{T}}$. The notation $\mathcal{E}_{\overrightarrow{\mathcal{T}}}$ also represents the edge set of this directed tree. 
Optimization B: This problem is as follows:

$$
\begin{array}{lll}
\underset{\overline{\mathbf{X}} \in \mathbb{F}_{m}}{\operatorname{minimize}} & \sum_{(i, j) \in \mathcal{E}_{\overrightarrow{\mathcal{T}}}} \operatorname{trace}\left\{\mathbf{T}_{i j} \overline{\mathbf{X}}_{i j}^{*}\right\} & \\
\text { subject to } & \overline{\mathbf{X}}_{k}=\overline{\mathbf{X}}_{k}^{\text {ref }} & k=1, \ldots,|\mathcal{T}|, \\
& \overline{\mathbf{X}} \succeq 0, &
\end{array}
$$

where $\mathbf{T}_{i j}$ 's are arbitrary constant matrices of appropriate dimensions and

$$
\overline{\mathbf{X}}_{k} \triangleq \overline{\mathbf{X}}\left(\bar{V}_{k}\right), \quad \overline{\mathbf{X}}_{k}^{\mathrm{ref}} \triangleq \overline{\mathbf{X}}^{\mathrm{ref}}\left(\bar{V}_{k}\right) \quad \text { and } \quad \overline{\mathbf{X}}_{i j} \triangleq \overline{\mathbf{X}}\left(\bar{V}_{i} \backslash \bar{V}_{j}, \bar{V}_{j} \backslash \bar{V}_{i}\right)
$$

for every $i, j, k \in\{1, \ldots,|\mathcal{T}|\}$.

Let $\overline{\mathbf{X}}^{\text {opt }} \in \mathbb{F}_{m}$ denote an arbitrary solution of problem $\left(5.32\right.$ and $\mathbf{X}^{\text {opt }} \in \mathbb{F}_{n}$ be equal to $\overline{\mathbf{X}}^{\mathrm{opt}}\left(\mathcal{V}_{\mathcal{G}}\right)$. Then, $\mathbf{X}^{\mathrm{opt}}$ is called the subsolution to Optimization B associated with $\overline{\mathbf{X}}^{\text {opt }}$. Note that $\mathbf{X}^{\text {opt }}$ and $\mathbf{X}^{\text {ref }}$ share the same diagonal and values for the entries corresponding to the edges of $\mathcal{G}$. Therefore, if $\mathbf{X}^{\text {ref }}$ is feasible for problem (5.27), then $\mathbf{X}^{\text {opt }}$ is feasible for (5.27) as well. Hence, Optimization B is a positive semidefinite matrix completion problem with the input $\mathbf{X}^{\text {ref }}$ and the output $\mathbf{X}^{\mathrm{opt}}$.

TheOREM 5.4. Given an arbitrary solution $\mathbf{X}^{\text {ref }}$ of the problem (5.27), every subsolution $\mathbf{X}^{\mathrm{opt}}$ of Optimization B is a solution to the LMI problem (5.27) and has the property

$$
\operatorname{rank}\left\{\mathbf{X}^{\mathrm{opt}}\right\}=\max \left\{\operatorname{rank}\left\{\mathbf{X}^{\mathrm{ref}}\left(V_{k}\right)\right\}|k=1, \ldots,| \mathcal{T} \mid\right\}
$$

provided that the following equality holds for every $(i, j) \in \mathcal{E}_{\overrightarrow{\mathcal{T}}}$ :

$$
\operatorname{rank}\left\{\mathbf{S}_{i j}^{+} \mathbf{T}_{i j} \mathbf{S}_{j i}^{+}\right\}=\min \left\{\operatorname{rank}\left\{\mathbf{S}_{i j}^{+}\right\}, \operatorname{rank}\left\{\mathbf{S}_{j i}^{+}\right\}\right\} .
$$

Proof. According to the constraints (5.32b) and (5.32c), if $\overline{\mathbf{X}}$ is a feasible point for (5.32), then $\overline{\mathbf{X}}\left(\mathcal{V}_{\mathcal{G}}\right)$ is feasible for the LMI problem (5.27) as well. Therefore, for the case $|\mathcal{T}|=2$, the proof follows immediately from Theorem 5.2 . To prove by induction in the general case, assume that the statement of Theorem 5.4 holds if $|\mathcal{T}| \leq p$ for an arbitrary natural number $p$, and the goal is to show its validity for $|\mathcal{T}|=p+1$. With no loss of generality, assume that $V_{p+1}$ is a leaf of $\overrightarrow{\mathcal{T}}$ and that $\left(V_{p}, V_{p+1}\right)$ is a directed edge of this tree. Consider a tree decomposition $\mathcal{T}^{\prime}=\left(\mathcal{V}_{\mathcal{T}^{\prime}}, \mathcal{E}_{\mathcal{T}}\right)$ for the sparsity graph of Optimization B with the bags $\bar{V}_{1}^{\prime}, \ldots, \bar{V}_{|\mathcal{T}|}^{\prime}$, where each bag $\bar{V}_{i}^{\prime}$ is defined as the union of $\bar{V}_{i}$ and its parent in the oriented tree $\mathcal{T}$, if any. It results from the chordal theorem that the constraint $\overline{\mathbf{X}} \succeq 0$ in Optimization B can be replaced by the set of constraints $\overline{\mathbf{X}}\left(\bar{V}_{j}^{\prime}\right) \succeq 0$ for $j=1, \ldots, p+1$. This implies that Optimization B can be decomposed into $p=|\mathcal{T}|-1$ independent semidefinite programs:

$$
\begin{array}{ll}
\underset{\overline{\mathbf{X}}\left(\bar{V}_{j}^{\prime}\right)}{\operatorname{minimize}} & \operatorname{trace}\left\{\mathbf{T}_{i j} \overline{\mathbf{X}}_{i j}^{*}\right\} \\
\text { subject to } & \overline{\mathbf{X}}_{i}=\overline{\mathbf{X}}_{i}^{\mathrm{ref}} \\
& \overline{\mathbf{X}}_{j}=\overline{\mathbf{X}}_{j}^{\mathrm{ref}} \\
& \overline{\mathbf{X}}\left(\bar{V}_{j}^{\prime}\right) \succeq 0,
\end{array}
$$


for every $(i, j) \in \mathcal{E}_{\overrightarrow{\mathcal{T}}}$. Notice that the submatrices $\overline{\mathbf{X}}_{1}^{\text {ref }}, \ldots, \overline{\mathbf{X}}_{|\mathcal{T}|}^{\text {ref }}$ all have the same rank $r$. By defining $\bar{V}_{0}^{\prime} \triangleq \bar{V}_{1}^{\prime} \cup \bar{V}_{2}^{\prime} \ldots \cup \bar{V}_{p}^{\prime}$, it follows from the induction assumption and the decomposition property of Optimization B that

$$
\operatorname{rank}\left\{\overline{\mathbf{X}}^{\mathrm{opt}}\left(\bar{V}_{0}^{\prime}\right)\right\}=\max \left\{\operatorname{rank}\left\{\overline{\mathbf{X}}_{k}^{\mathrm{ref}}\right\} \mid k=1, \ldots, p\right\}=r .
$$

Now, consider the block matrix

$$
\mathbf{M}(\mathbf{U}) \triangleq\left[\begin{array}{ccc}
\overline{\mathbf{X}}^{\mathrm{opt}}\left({\overline{V_{0}}}_{0}^{\prime} \cap{\overline{V^{\prime}}}_{|\mathcal{T}|}^{\prime}\right) & \overline{\mathbf{X}}^{\mathrm{opt}}\left(\bar{V}_{0}^{\prime} \cap \bar{V}_{|\mathcal{T}|}^{\prime}, \bar{V}_{0}^{\prime} \backslash \bar{V}_{|\mathcal{T}|}^{\prime}\right) & \overline{\mathbf{X}}^{\mathrm{opt}}\left(\bar{V}_{0}^{\prime} \cap \bar{V}_{|\mathcal{T}|}^{\prime}, \bar{V}_{|\mathcal{T}|}^{\prime} \backslash \bar{V}_{0}^{\prime}\right) \\
\overline{\mathbf{X}}^{\mathrm{opt}}\left(\bar{V}_{0}^{\prime} \backslash \bar{V}_{|\mathcal{T}|}^{\prime}, \bar{V}_{0}^{\prime} \cap \bar{V}_{|\mathcal{T}|}^{\prime}\right) & \overline{\mathbf{X}}^{\mathrm{opt}}\left(\bar{V}_{0}^{\prime} \backslash \bar{V}_{|\mathcal{T}|}^{\prime}\right) & \mathbf{\mathbf { X }}^{\mathrm{opt}}\left(\bar{V}_{|\mathcal{T}|}^{\prime} \backslash \bar{V}_{0}^{\prime}, \bar{V}_{0}^{\prime} \cap \bar{V}_{|\mathcal{T}|}^{\prime}\right) \\
\mathbf{U}^{*} & \mathbf{U} & \overline{\mathbf{X}}^{\mathrm{opt}}\left({\overline{V^{\prime}}}_{|\mathcal{T}|}^{\prime} \backslash \bar{V}_{0}^{\prime}\right)
\end{array}\right] .
$$

According to Theorem 5.2 , it only remains to prove that

$$
\operatorname{rank}\left\{\mathbf{S}_{|\mathcal{T}|, 0}^{+} \mathbf{T}_{|\mathcal{T}|, 0} \mathbf{S}_{0,|\mathcal{T}|}^{+}\right\}=\min \left\{\operatorname{rank}\left\{\mathbf{S}_{|\mathcal{T}|, 0}^{+}\right\}, \operatorname{rank}\left\{\mathbf{S}_{0,|\mathcal{T}|}^{+}\right\}\right\}
$$

where

$$
\mathbf{T}_{|\mathcal{T}|, 0}=\left[\begin{array}{ll}
\mathbf{0} & \left.\mathbf{T}_{|\mathcal{T}|-1,|\mathcal{T}|}\right]
\end{array}\right.
$$

One can write

$$
\begin{aligned}
& \operatorname{rank}\left\{\mathbf{S}_{|\mathcal{T}|, 0}^{+}\left[\mathbf{0} \quad \mathbf{T}_{|\mathcal{T}|-1,|\mathcal{T}|}\right] \mathbf{S}_{0,|\mathcal{T}|}^{+}\right\}=\operatorname{rank}\left\{\mathbf{S}_{|\mathcal{T}|-1,|\mathcal{T}|}^{+} \mathbf{T}_{|\mathcal{T}|-1,|\mathcal{T}|} \mathbf{S}_{|\mathcal{T}|,|\mathcal{T}|-1}^{+}\right\} \\
& =\min \left\{\operatorname{rank}\left\{\mathbf{S}_{|\mathcal{T}|-1,|\mathcal{T}|}^{+}\right\}, \operatorname{rank}\left\{\mathbf{S}_{|\mathcal{T}|,|\mathcal{T}|-1}^{+}\right\}\right\} \\
& =\operatorname{rank}\left\{\mathbf{S}_{|\mathcal{T}|-1,|\mathcal{T}|}^{+}\right\} \\
& =\operatorname{rank}\left\{\mathbf{S}_{0,|\mathcal{T}|}^{+}\right\} \\
& =\min \left\{\operatorname{rank}\left\{\mathbf{S}_{|\mathcal{T}|, 0}^{+}\right\}, \operatorname{rank}\left\{\mathbf{S}_{0,|\mathcal{T}|}^{+}\right\}\right\} .
\end{aligned}
$$

This completes the proof.

Note that the condition 5.35 required in Theorem 5.4 is satisfied for generic choices of $\mathbf{T}_{i j}$.

EXAMPLE 4. Consider a tree decomposition $\mathcal{T}$ with three bags $V_{1}=\{1,2,3\}$, $V_{2}=\{3,4,5\}$ and $V_{3}=\{5,6,7\}$, and the edge set $\mathcal{E}_{\mathcal{T}}=\left\{\left(V_{1}, V_{2}\right),\left(V_{2}, V_{3}\right)\right\}$. Suppose that the partially known matrix solution is as follows:

$\mathbf{X}^{\text {ref }}=\left[\begin{array}{ccc|cccc}\hline 2 & 1 & 1 & u_{11}^{*} & u_{21}^{*} & w_{11}^{*} & w_{21}^{*} \\ 1 & 1 & 1 & u_{12}^{*} & u_{22}^{*} & w_{12}^{*} & w_{22}^{*} \\ 1 & 1 & 1 & 1 & 1 & v_{11}^{*} & v_{21}^{*} \\\right.$\cline { 2 - 6 }$\left.u_{11} & u_{12} & 1 & 1 & 1 & v_{12}^{*} & v_{22}^{*} \\ u_{21} & u_{22} & 1 & 1 & 1 & 1 & 1 \\ w_{11} & w_{12} & v_{11} & v_{12} & 1 & 2 & 1 \\ w_{21} & w_{22} & v_{21} & v_{22} & 1 & 1 & 3\end{array}\right]$.

It can be verified that

$$
\operatorname{rank}\left\{\mathbf{X}^{\mathrm{ref}}\left(V_{1}\right)\right\}=2, \quad \operatorname{rank}\left\{\mathbf{X}^{\mathrm{ref}}\left(V_{2}\right)\right\}=1, \quad \operatorname{rank}\left\{\mathbf{X}^{\mathrm{ref}}\left(V_{3}\right)\right\}=3,
$$

and that there exists only one unique solution for each unknown block

$$
\mathbf{X}_{12}=\left[\begin{array}{ll}
u_{11} & u_{12} \\
u_{21} & u_{22}
\end{array}\right] \quad \text { and } \quad \mathbf{X}_{23}=\left[\begin{array}{ll}
v_{11} & v_{12} \\
v_{21} & v_{22}
\end{array}\right]
$$


to meet the constraint $\mathbf{X} \succeq 0$. Hence, the only freedom for the matrix completion problem is on the choice of the remaining block

$$
\left[\begin{array}{ll}
w_{11} & w_{12} \\
w_{21} & w_{22}
\end{array}\right]
$$

Therefore, optimization problems solved over the blocks $\mathbf{X}_{12}$ and $\mathbf{X}_{23}$ would not result in a rank-3 solution. To resolve the issue, we enrich $\mathbf{X}^{\text {ref }}$ to obtain a matrix $\overline{\mathbf{X}}^{\text {ref }}$ by adding multiple rows and columns to $\mathbf{X}^{\text {ref }}$ in order to make the ranks of all resulting bags equal:

$$
\overline{\mathbf{X}}^{\text {ref }}=\left[\begin{array}{cccc|cccccc}
\hline 1 & 0 & 0 & 0 & u_{11}^{*} & u_{21}^{*} & u_{31}^{*} & u_{41}^{*} & w_{11}^{*} & w_{21}^{*} \\
0 & 2 & 1 & 1 & u_{12}^{*} & u_{22}^{*} & u_{32}^{*} & u_{42}^{*} & w_{12}^{*} & w_{22}^{*} \\
0 & 1 & 1 & 1 & u_{13}^{*} & u_{23}^{*} & u_{33}^{*} & u_{43}^{*} & w_{13}^{*} & w_{23}^{*} \\
0 & 1 & 1 & 1 & 0 & 0 & 1 & 1 & v_{11}^{*} & v_{12}^{*} \\
\cline { 1 - 8 } u_{11} & u_{12} & u_{13} & 0 & 1 & 0 & 0 & 0 & v_{12}^{*} & v_{22}^{*} \\
u_{21} & u_{22} & u_{23} & 0 & 0 & 1 & 0 & 0 & v_{13}^{*} & v_{23}^{*} \\
u_{31} & u_{32} & u_{33} & 1 & 0 & 0 & 1 & 1 & v_{14}^{*} & v_{24}^{*} \\
u_{41} & u_{42} & u_{43} & 1 & 0 & 0 & 1 & 1 & 1 & 1 \\
w_{11} & w_{12} & w_{13} & v_{11} & v_{12} & v_{13} & v_{14} & 1 & 2 & 1 \\
w_{21} & w_{22} & w_{23} & v_{21} & v_{22} & v_{23} & v_{24} & 1 & 1 & 3
\end{array}\right] .
$$

Now, we have

$$
\bar{V}_{1}=\{1,2,3,4\}, \quad \bar{V}_{2}=\{4,5,6,7,8\}, \quad \text { and } \quad \bar{V}_{3}=\{8,9,10\}
$$

and

$$
\operatorname{rank}\left\{\overline{\mathbf{X}}_{1}^{\mathrm{ref}}\right\}=3, \quad \operatorname{rank}\left\{\overline{\mathbf{X}}_{2}^{\mathrm{ref}}\right\}=3, \quad \text { and } \quad \operatorname{rank}\left\{\overline{\mathbf{X}}_{3}^{\mathrm{ref}}\right\}=3 .
$$

Therefore, the conditions of Theorem 5.4 hold for generic constant matrices $\mathbf{T}_{12}$ and $\mathbf{T}_{23}$. As a result, every solution $\overline{\mathbf{X}}$ of Optimization B has the property

$$
\operatorname{rank}\left\{\overline{\mathbf{X}}^{\text {opt }}\right\}=3 \text {. }
$$

As a final step, the deletion of those rows and columns of $\overline{\mathbf{X}}^{\text {opt }}$ with indices 1,5 and 6 yields a completion of $\mathbf{X}^{\text {ref }}$ with rank 3.

6. Low-Rank Solutions via Complex Analysis. Consider the problem of finding a low-rank solution $\mathrm{X}^{\mathrm{opt}}$ for the LMI problem 4.5). Theorem 4.2 can be used for this purpose, but it needs solving one of the following graph problems: (i) designing a supergraph $\mathcal{G}^{\prime}$ minimizing the upper bound given in 4.6), or (ii) obtaining a tree decomposition of $\mathcal{G}$ with the minimum width. Although these graph problems are easy to solve for highly sparse and structured graphs, they are NP-hard for arbitrary graphs. A question arises as to whether a low-rank solution can be obtained using a polynomial-time algorithm without requiring an expensive graph analysis. This problem will be addressed in this section.

Definition 6.1. Given a complex number $z$, define

$$
z^{\text {ray }} \triangleq\{\lambda z \mid \lambda \in \mathbb{R}, \lambda \geq 0\} .
$$

Definition 6.2. A finite set $U \subset \mathbb{C}$ is called sign-definite in $\mathbb{C}$ if $U$ and $-U$ can be separated in the complex plane by a line passing through the origin, where 
$-U \triangleq\{-u \mid u \in U\}[5]$. Moreover, a finite set $U \subset \mathbb{R}$ is called sign-definite in $\mathbb{R}$ if its members are all nonnegative or all nonpositive.

Optimization C: Let $\mathcal{G}$ be a simple graph with $n$ vertices and $\mathbb{F}$ be equal to either $\mathbb{R}$ or $\mathbb{C}$. Consider arbitrary matrices $\mathbf{X}^{\mathrm{ref}} \in \mathbb{F}_{n}^{+}$and $\mathbf{T} \in \mathbb{F}_{n}$ such that $\mathscr{G}(\mathbf{T})$ is a supergraph of $\mathcal{G}$. The problem

$$
\begin{array}{clr}
\underset{\mathbf{X} \in \mathbb{F}_{n}}{\operatorname{minimize}} & \operatorname{trace}\{\mathbf{T X}\} & \\
\text { subject to } & X_{k k}=X_{k k}^{\mathrm{ref}} & k \in \mathcal{V}_{\mathcal{G}}, \\
& X_{i j}-X_{i j}^{\mathrm{ref}} \in T_{i j}^{\mathrm{ray}} & (i, j) \in \mathcal{E}_{\mathcal{G}}, \\
& \mathbf{X} \succeq 0, &
\end{array}
$$

is referred to as "Optimization $\mathrm{C}$ with the input $\left(\mathcal{G}, \mathbf{X}^{\mathrm{ref}}, \mathbf{T}, \mathbb{F}\right)$ ".

Lemma 6.3. Assume that $\mathbf{X}^{\text {ref }}$ is positive definite. Every solution $\mathbf{X}^{\mathrm{opt}}$ of Optimization $C$ with the input $\left(\mathcal{G}, \mathbf{X}^{\mathrm{ref}}, \mathbf{T}, \mathbb{H}\right)$ satisfies the inequality

$$
\operatorname{rank}\left\{\mathbf{X}^{\mathrm{opt}}\right\} \leq n-\operatorname{msr}_{\mathbb{H}}(\mathscr{G}(\mathbf{T})) .
$$

Proof. Constraints $(6.2 \mathrm{~b})$ and $(6.2 \mathrm{c})$ imply that for any feasible matrix $\mathbf{X}$, the matrix $\mathbf{X}-\mathbf{X}^{\text {ref }}$ belongs to the convex cone

$$
C=\left\{\mathbf{W} \in \mathbb{F}_{n} \mid W_{k k}=0 \quad \text { for } \quad k \in \mathcal{V}_{\mathcal{G}}, \quad W_{i j} \in T_{i j}^{\mathrm{ray}} \quad \text { for } \quad(i, j) \in \mathcal{E}_{\mathcal{G}}\right\} .
$$

Hence, the dual matrix variable $\boldsymbol{\Lambda}$ is a member of the dual cone

$$
C^{\perp}=\left\{\mathbf{W} \in \mathbb{F}_{n} \mid \operatorname{Re}\left\{W_{i j} T_{i j}^{*}\right\} \geq 0 \text { for }(i, j) \in \mathcal{E}_{\mathcal{G}}, W_{i j}=0 \text { for }(i, j) \notin \mathcal{E}_{\mathcal{G}} \text { and } \mathrm{i} \neq \mathrm{j}\right\} .
$$

Therefore, the Lagrangian is equal to

$$
\begin{aligned}
\mathcal{L}(\mathbf{X}, \boldsymbol{\Lambda}, \boldsymbol{\Phi}) & =\operatorname{trace}\{\mathbf{T X}\}+\operatorname{trace}\left\{\boldsymbol{\Lambda}\left(\mathbf{X}-\mathbf{X}^{\mathrm{ref}}\right)\right\}-\operatorname{trace}\{\boldsymbol{\Phi} \mathbf{X}\} \\
& =\operatorname{trace}\{(\boldsymbol{\Lambda}+\mathbf{T}-\mathbf{\Phi}) \mathbf{X}\}-\operatorname{trace}\left\{\boldsymbol{\Lambda} \mathbf{X}^{\mathrm{ref}}\right\},
\end{aligned}
$$

where $\mathbf{\Phi} \succeq 0$ denotes the matrix dual variable corresponding to the constraint $\mathbf{X} \succeq 0$. The infimum of the Lagrangian over $\mathbf{X}$ is $-\infty$ unless $\boldsymbol{\Phi}=\boldsymbol{\Lambda}+\mathbf{T}$. Therefore, the dual problem is as follows:

$$
\begin{array}{llr}
\underset{\Lambda \in \mathbb{H}_{n}}{\operatorname{maximize}} & -\operatorname{trace}\left\{\boldsymbol{\Lambda} \mathbf{X}^{\text {ref }}\right\} & \\
\text { subject to } & \operatorname{Re}\left\{\Lambda_{i j} T_{i j}^{*}\right\} \geq 0 & \quad(i, j) \in \mathcal{E}_{\mathcal{G}} \\
& \Lambda_{i j}=0, & (i, j) \notin \mathcal{E}_{\mathcal{G}} \text { and } \mathrm{i} \neq \mathrm{j} \\
& \boldsymbol{\Lambda}+\mathbf{T} \succeq 0 . &
\end{array}
$$

By pushing the diagonal entries of $\boldsymbol{\Lambda}$ toward infinity, the inequality $\boldsymbol{\Lambda}+\mathbf{T} \succeq 0$ will become strict. Hence, strong duality holds according to the Slater's condition. Let $\boldsymbol{\Phi}=\boldsymbol{\Phi}^{\text {opt }}$ denote an arbitrary dual solution. The complementary slackness condition $\operatorname{trace}\left\{\boldsymbol{\Phi}^{\mathrm{opt}} \mathbf{X}^{\mathrm{opt}}\right\}=0$ yields that

$$
\operatorname{rank}\left\{\boldsymbol{\Phi}^{\mathrm{opt}}\right\}+\operatorname{rank}\left\{\mathbf{X}^{\mathrm{opt}}\right\} \leq n .
$$

On the other hand, it can be deduced from the equation $\mathbf{\Phi}=\boldsymbol{\Lambda}+\mathbf{T}$ together with $6.6 \mathrm{~b}$ ) and $6.6 \mathrm{c}$ that

$$
\mathscr{G}(\mathbf{T})=\mathscr{G}\left(\mathbf{\Phi}^{\mathrm{opt}}\right) .
$$


Now, combining (6.7) and 6.8 completes the proof.

TheOREm 6.4. Assume that $\mathbf{M}_{1}, \ldots, \mathbf{M}_{p}$ are arbitrary matrices in $\mathbb{S}_{n}$. Suppose that $a_{1}, \ldots, a_{p}$ are real numbers such that the LMI problem

$$
\text { find } \quad \mathbf{X} \in \mathbb{S}_{n}
$$

subject to $\quad \operatorname{trace}\left\{\mathbf{M}_{k} \mathbf{X}\right\} \leq a_{k} \quad k=1, \ldots, p$,

$$
\mathbf{X} \succeq 0
$$

has a positive-definite feasible solution $\mathbf{X}^{\text {ref }} \in \mathbb{S}_{n}$. Let $\mathbf{T} \in \mathbb{H}_{n}$ be an arbitrary matrix such that $\operatorname{Re}\{\mathbf{T}\}=0_{n \times n}$ and $\mathscr{G}(\mathbf{T})$ is a supergraph of $\mathscr{G}\left(\mathbf{M}_{1}\right) \cup \cdots \cup \mathscr{G}\left(\mathbf{M}_{p}\right)$.

a) Every solution $\mathbf{X}^{\mathrm{opt}} \in \mathbb{H}_{n}$ of Optimization $C$ with the input $\left(\mathcal{G}, \mathbf{X}^{\mathrm{ref}}, \mathbf{T}, \mathbb{H}\right)$ is a solution of the LMI problem (6.9) and satisfies the relations

$$
\begin{aligned}
& \operatorname{rank}\left\{\mathbf{X}^{\mathrm{opt}}\right\} \leq n-\operatorname{msr}_{\mathbb{H}}(\mathscr{G}(\mathbf{T})), \\
& \operatorname{rank}\left\{\mathbf{X}^{\mathrm{opt}}\right\} \leq \mathrm{Z}^{+}(\mathscr{G}(\mathbf{T})) .
\end{aligned}
$$

b) The matrix $\operatorname{Re}\left\{\mathbf{X}^{\mathrm{opt}}\right\}$ is a real-valued solution of the LMI problem (6.9) and satisfies the inequalities

$$
\begin{aligned}
& \operatorname{rank}\left\{\mathbf{X}^{\text {real }}\right\} \leq \min \left\{2\left(n-\operatorname{msr}_{\mathbb{H}}(\mathscr{G}(\mathbf{T})), n\right\},\right. \\
& \operatorname{rank}\left\{\mathbf{X}^{\text {real }}\right\} \leq \min \left\{2 \mathrm{Z}^{+}(\mathscr{G}(\mathbf{T})), n\right\} .
\end{aligned}
$$

Proof. For every feasible solution $\mathbf{X}$ of Optimization C, we have

$$
\operatorname{trace}\left\{\mathbf{M}_{\mathrm{k}} \mathbf{X}\right\}=\operatorname{trace}\left\{\mathbf{M}_{\mathrm{k}} \mathbf{X}^{\mathrm{ref}}\right\} \quad \text { for } \mathrm{k}=1, \ldots, \mathrm{p} .
$$

Hence, every feasible solution of Optimization C is a solution of the LMI problem 6.9p as well. Now, the proof of Part (a) follows from Lemma 6 For Part (b), it is straightforward to verify that $\mathbf{X}^{\text {real }}$ defined as $\frac{1}{2}\left(\mathbf{X}^{\mathrm{opt}}+\left(\mathbf{X}^{\mathrm{opt}}\right)^{\mathrm{T}}\right)$ is a feasible solution of 6.9. Moreover,

$$
\begin{aligned}
\operatorname{rank}\left\{\mathbf{X}^{\text {real }}\right\} & \leq \operatorname{rank}\left\{\mathbf{X}^{\mathrm{opt}}\right\}+\operatorname{rank}\left\{\left(\mathbf{X}^{\mathrm{opt}}\right)^{\mathrm{T}}\right\} \\
& =2 \operatorname{rank}\left\{\mathbf{X}^{\mathrm{opt}}\right\} .
\end{aligned}
$$

The proof follows from the above inequality and Part (a).

Consider an LMI problem with real-valued coefficients. Theorem 6.4 states that the complex-valued Optimization C can be exploited to find a real solution of the LMI problem under study with a guaranteed bound on its rank. This bound might be looser than the ones derived in Theorem 4.2, but is still small for very sparse graphs. Note that although the calculation of the bound given in (6.11a) is an NP-hard problem, Optimization $\mathrm{C}$ is polynomial-time solvable without requiring any expensive graph preprocessing. In what follows, we improve the bound obtained in Theorem 6.4 for a structured LMI problem.

Lemma 6.5. Let $U=\left\{u_{1}, \ldots, u_{n}\right\} \subset \mathbb{F}$ be sign-definite in $\mathbb{F}$. Then, the set

$$
\angle U \triangleq\left\{x \in \mathbb{F} \mid \operatorname{Re}\left\{u_{k} x\right\} \leq 0 \text { for } k=1, \ldots, n\right\}
$$

forms a non-trivial convex cone in $\mathbb{F}$.

Proof. In the case $\mathbb{F}=\mathbb{R}$, the set $U$ is either the ray of nonnegative real numbers or non-positive real numbers. Hence, $U$ is a non-trivial convex cone if $\mathbb{F}=\mathbb{R}$. Consider now the case $\mathbb{F}=\mathbb{C}$. The convexity of $\angle U$ results from the fact that this set is 
described by linear inequalities. $\angle U$ is also a cone because $\lambda x \in \angle U$ for every $x \in \angle U$ and $\lambda \geq 0$. On the other hand, by the definition of a sign-definite set, there exists a line passing through the origin that separates the sets $\left\{u_{1}, \ldots, u_{n}\right\}$ and $\left\{-u_{1}, \ldots,-u_{n}\right\}$. Assume that this line makes the angle $\alpha$ with the real axis. Then, one of the two points $\exp \left[\left(\frac{\pi}{2}+\alpha\right) \mathbf{i}\right]$ and $\exp \left[\left(-\frac{\pi}{2}+\alpha\right) \mathbf{i}\right]$ belongs to $\angle U$. As a result, $\angle U$ is non-trivial.

By leveraging the result of Lemma 6 , the bound proposed in Theorem 6.4 will be improved for a sign-definite LMI problem below.

TheOrem 6.6. Assume that $\mathbf{M}_{1}, \ldots, \mathbf{M}_{p}$ belong to the set $\mathbb{F}_{n}$ that is equal to either $\mathbb{S}_{n}$ or $\mathbb{H}_{n}$. Let $a_{1}, \ldots, a_{m}$ be real numbers such that the LMI problem

$$
\begin{array}{ll}
\text { find } & \mathbf{X} \in \mathbb{F}_{n} \\
\text { subject to } & \operatorname{trace}\left\{\mathbf{M}_{k} \mathbf{X}\right\} \leq a_{k} \quad k=1, \ldots, p, \\
& \mathbf{X} \succeq 0,
\end{array}
$$

has a positive-definite feasible solution $\mathbf{X}^{\mathrm{ref}} \in \mathbb{F}_{n}^{+}$. Let $\mathcal{G}=\mathscr{G}\left(\mathbf{M}_{1}\right) \cup \cdots \cup \mathscr{G}\left(\mathbf{M}_{p}\right)$ and suppose that the set $\mathscr{M}_{i j}$ composed of the $(i, j)$ entries of $\mathbf{M}_{1}, \ldots, \mathbf{M}_{p}$ is sign-definite for every pair $(i, j) \in \mathcal{E}_{\mathcal{G}}$. Consider a matrix $\mathbf{T} \in \mathbb{F}_{n}$ such that $\mathscr{G}(\mathbf{T})$ is a supergraph of $\mathcal{G}$ and that $T_{i j} \in \angle \mathcal{M}_{i j}$ for every $(i, j) \in \mathcal{E}_{\mathcal{G}}$. Then, every solution $\mathbf{X}^{\mathrm{opt}} \in \mathbb{F}_{n}$ of Optimization $C$ with the input $\left(\mathcal{G}, \mathbf{X}^{\mathrm{ref}}, \mathbf{T}, \mathbb{F}\right)$ is a solution of the LMI problem 6.15) and satisfies the inequalities

$$
\begin{aligned}
& \operatorname{rank}\left\{\mathbf{X}^{\mathrm{opt}}\right\} \leq n-\operatorname{msr}_{\mathbb{F}}(\mathscr{G}(\mathbf{T})), \\
& \operatorname{rank}\left\{\mathbf{X}^{\mathrm{opt}}\right\} \leq \mathrm{Z}^{+}(\mathscr{G}(\mathbf{T}))
\end{aligned}
$$

Proof. According to Lemma 6, a matrix $\mathbf{T}$ with the properties mentioned in the theorem always exists. We have $X_{i j}^{\text {opt }}-X_{i j}^{\text {ref }} \in T_{i j}^{\text {ray }} \subseteq \angle \mathcal{M}_{i j}$ for every $(i, j) \in \mathcal{E}_{\mathcal{G}}$. Hence, for $k=1, \ldots, p$, one can write

$$
\operatorname{Re}\left\{\mathbf{M}_{k}(i, j)\left(X_{i j}^{\mathrm{opt}}-X_{i j}^{\mathrm{ref}}\right)\right\} \leq 0
$$

or equivalently

$$
\operatorname{trace}\left\{\mathbf{M}_{\mathrm{k}} \mathbf{X}^{\mathrm{opt}}\right\} \leq \operatorname{trace}\left\{\mathbf{M}_{\mathrm{k}} \mathbf{X}^{\mathrm{ref}}\right\}
$$

$\left(\mathbf{M}_{k}(i, j)\right.$ denotes the $(i, j)$ entry of $\left.\mathbf{M}_{k}\right)$. Consequently, $\mathbf{X}^{\text {opt }}$ is a feasible solution of the LMI problem 6.15) and satisfies the inequalities (6.16a and 6.16b) in light of Lemma 6,

Theorem 6.6 improves upon the results of Theorem 6.4 for structured LMI problems in two directions: (i) extension to the complex case, and (ii) reduction of the upper bound by a factor of 2 in the real case.

7. Low-Rank Solutions for Affine Problems. In this section, we will generalize the results derived earlier to the affine rank minimization problem.

DEFINITION 7.1. For an arbitrary matrix $\mathbf{W} \in \mathbb{C}^{m \times r}$, the notation $\mathscr{B}(\mathbf{W})=$ $\left(\mathcal{V}_{\mathcal{B}}, \mathcal{E}_{\mathcal{B}}\right)$ denotes a bipartite graph defined as:

1. $\mathcal{V}_{\mathcal{B}}$ is the union of the first vertex set $\mathcal{V}_{\mathcal{B}_{1}}=\{1, \ldots, n\}$ and the second set vertex set $\mathcal{V}_{\mathcal{B}_{2}}=\{1, \ldots, m\}$, associated with the two parts of the graph.

2. For every $(i, j) \in \mathcal{V}_{\mathcal{B}_{1}} \times \mathcal{V}_{\mathcal{B}_{2}}$, we have $(i, j) \in \mathcal{E}_{\mathcal{B}}$ if and only if $W_{i j} \neq 0$.

DeFINITION 7.2. Consider an arbitrary matrix $\mathbf{X} \in \mathbb{H}_{n}$ and two natural numbers $m$ and $r$ such that $n \geq m+r$. The matrix $\operatorname{sub}_{m, r}\{\mathbf{X}\}$ is defined as the $m \times r$ submatrix 
of $\mathbf{X}$ corresponding to the first $m$ rows and the last $r$ columns of the $(m+r)$-th leading principal submatrix of $\mathbf{X}$.

THEOREM 7.3. Consider the feasibility problem

$$
\text { find } \quad \mathbf{W} \in \mathbb{R}^{m \times r}
$$

subject to $\quad \operatorname{trace}\left\{\mathbf{N}_{\mathrm{k}} \mathbf{W}\right\} \leq \mathrm{a}_{\mathrm{k}} \quad k=1, \ldots, p$,

where $a_{1}, \ldots, a_{p} \in \mathbb{R}$ and $\mathbf{N}_{1}, \ldots, \mathbf{N}_{p} \in \mathbb{R}^{r \times m}$. Let $\mathbf{W}^{\text {ref }} \in \mathbb{R}^{m \times r}$ denote a feasible solution of this feasibility problem and $\mathbf{X}^{\mathrm{ref}} \in \mathbb{S}_{r+m}^{+}$be a matrix such that $\operatorname{sub}_{r, m}\left\{\mathbf{X}^{\mathrm{ref}}\right\}=\mathbf{W}^{\mathrm{ref}}$. Define $\mathcal{G}=\mathscr{B}\left(\mathbf{N}_{1}^{\mathrm{T}}\right) \cup \cdots \cup \mathscr{B}\left(\mathbf{N}_{p}^{\mathrm{T}}\right)$. The following statements hold:

a) Consider an arbitrary supergraph $\mathcal{G}^{\prime}$ of $\mathcal{G}$ with $n$ vertices, where $n \geq r+$ $m$. Let $\overline{\mathbf{X}}^{\text {opt }}$ denote an arbitrary solution of Optimization $A$ with the input $\left(\mathcal{G}, \mathcal{G}^{\prime}, \mathbf{T}, \mathbf{X}^{\mathrm{ref}}\right)$. Then, $\mathbf{W}^{\mathrm{opt}}$ defined as $\operatorname{sub}_{m, r}\left\{\overline{\mathbf{X}}^{\mathrm{opt}}\right\}$ is a solution of the feasibility problem (7.1) and satisfies the relations

$$
\begin{aligned}
& \operatorname{rank}\left\{\mathbf{W}^{\text {opt }}\right\} \leq\left|\mathcal{G}^{\prime}\right|-\min \left\{\operatorname{msr}_{\mathbb{S}}\left(\mathcal{G}_{s}\right) \mid\left(\mathcal{G}^{\prime} \lambda \mathcal{G}\right) \subseteq \mathcal{G}_{s} \subseteq \mathcal{G}^{\prime}\right\} \\
& \operatorname{rank}\left\{\mathbf{W}^{\text {opt }}\right\} \leq \max \left\{\mathrm{Z}^{+}\left(\mathcal{G}_{s}\right) \mid\left(\mathcal{G}^{\prime} \lambda \mathcal{G}\right) \subseteq \mathcal{G}_{s} \subseteq \mathcal{G}^{\prime}\right\}
\end{aligned}
$$

b) Consider an arbitrary tree decomposition $\mathcal{T}$ of $\mathcal{G}$ with widtht. If $\mathcal{G}^{\prime}$ in Part (a) is considered as an enriched supergraph of $\mathcal{G}$ derived by $\mathcal{T}$, then

$$
\operatorname{rank}\left\{\mathbf{W}^{\mathrm{opt}}\right\} \leq t+1
$$

c) Let $\mathbf{X}^{\mathrm{opt}}$ denote an arbitrary solution of Optimization $C$ with the input $\left(\mathcal{G}, \mathbf{X}^{\mathrm{ref}}\right.$, $\mathbf{T}, \mathbb{H})$. Then, $\mathbf{W}^{\text {real }}$ defined as $\operatorname{sub}_{m, r}\left\{\operatorname{Re}\left\{\mathbf{X}^{\mathrm{opt}}\right\}\right\}$ is a solution of the feasibility problem 7.1 and satisfies the relations

$$
\begin{aligned}
& \operatorname{rank}\left\{\mathbf{W}^{\text {real }}\right\} \leq \min \left\{2\left(r+m-\operatorname{msr}_{\mathbb{H}}(\mathscr{G}(\mathbf{T})), r, m\right\}\right. \\
& \operatorname{rank}\left\{\mathbf{W}^{\text {real }}\right\} \leq \min \left\{2 \mathrm{Z}^{+}(\mathscr{G}(\mathbf{T})), r, m\right\} .
\end{aligned}
$$

Proof. The proof follows directly from Theorems 4.2 and 6.4 the conversion technique delineated in Subsection 1.3, and the inequality

$$
\operatorname{rank}\left\{\operatorname{sub}_{m, r}\{\mathbf{X}\}\right\} \leq \operatorname{rank}\{\mathbf{X}\}
$$

for every $\mathbf{X} \in \mathbb{S}_{n}$.

The following corollary is an immediate consequence of Theorem 7 .

COROLlary 7.4. If the feasibility problem 7.1 has a non-empty feasible set, then it has a solution $\mathbf{W}^{\text {opt }}$ with rank at most $\mathrm{tw}\left(\mathscr{B}\left(\mathbf{N}_{1}^{\mathrm{T}}\right) \cup \cdots \cup \mathscr{B}\left(\mathbf{N}_{p}^{\mathrm{T}}\right)\right)+1$.

As discussed in Subsection 1.3 the nuclear norm method is a popular technique for the minimum-rank matrix completion problem. In what follows, we adapt Theorem 7 to improve upon the nuclear norm method by incorporating a weighted sum into this norm and then obtain a guaranteed bound on the rank of every solution of the underlying convex optimization.

THEOREM 7.5. Suppose that $\mathcal{B}$ is a bipartite graph with bipartition $\left(\mathcal{V}_{1}, \mathcal{V}_{2}\right)$, such that $\left|\mathcal{V}_{1}\right|=m$ and $\left|\mathcal{V}_{2}\right|=r$. Given arbitrary matrices $\mathbf{W}^{\text {ref }}$ and $\mathbf{Q}$ in $\mathbb{R}^{m \times r}$, consider the convex program

$$
\begin{aligned}
& \underset{\mathbf{W} \in \mathbb{R}^{m \times r}}{\operatorname{minimize}} \quad\|\mathbf{W}\|_{*}+\operatorname{trace}\left\{\mathbf{Q}^{\mathrm{T}} \mathbf{W}\right\} \\
& \text { subject to } \quad W_{i j}=W_{i j}^{\text {ref }} \quad(i, j) \in \mathcal{E}_{\mathcal{B}} \text {. }
\end{aligned}
$$


Let $\mathcal{B}^{\prime}$ be defined as the supergraph $\mathcal{B} \cup \mathscr{B}(\mathbf{Q})$. Then, every solution $\mathbf{W}^{\mathrm{opt}}$ of the optimization (7.6) satisfies the inequalities

$$
\begin{aligned}
\operatorname{rank}\left\{\mathbf{W}^{\mathrm{opt}}\right\} & \leq m+r-\min \left\{\operatorname{msr}_{\mathbb{S}}\left(\mathcal{B}_{s}\right) \mid\left(\mathcal{B}^{\prime} \backslash \mathcal{B}\right) \subseteq \mathcal{B}_{s} \subseteq \mathcal{B}^{\prime}\right\} \\
\operatorname{rank}\left\{\mathbf{W}^{\text {opt }}\right\} & \leq \max \left\{\mathrm{Z}^{+}\left(\mathcal{B}_{s}\right) \mid\left(\mathcal{B}^{\prime} \lambda \mathcal{B}\right) \subseteq \mathcal{B}_{s} \subseteq \mathcal{B}^{\prime}\right\}
\end{aligned}
$$

Proof. Consider an arbitrary matrix $\mathbf{W} \in \mathbb{R}^{m \times r}$. It has been shown in [4] that the nuclear norm of $\mathbf{W}$ is equal to the optimal objective value of the optimization

$$
\begin{array}{ll}
\underset{\substack{\mathbf{X}_{1} \in \mathbb{R}^{m \times m} \\
\mathbf{X}_{2} \in \mathbb{R}^{r \times r}}}{\operatorname{minimize}} & \frac{1}{2} \operatorname{trace}\left\{\mathbf{X}_{1}\right\}+\frac{1}{2} \operatorname{trace}\left\{\mathbf{X}_{2}\right\} \\
\text { subject to } & {\left[\begin{array}{cc}
\mathbf{X}_{1} & \mathbf{W} \\
\mathbf{W}^{\mathrm{T}} & \mathbf{X}_{2}
\end{array}\right] \succeq 0 .}
\end{array}
$$

This implies that Optimization 7.6 is equivalent to

$$
\begin{array}{ll}
\underset{\substack{\mathbf{X}_{1} \in \mathbb{R}^{m \times m} \\
\mathbf{X}_{2} \in \mathbb{R}^{r \times r} \\
\mathbf{W} \in \mathbb{R}^{m \times r}}}{\operatorname{minimize}} & \frac{1}{2} \operatorname{trace}\left\{\mathbf{X}_{1}\right\}+\frac{1}{2} \operatorname{trace}\left\{\mathbf{X}_{2}\right\}+\operatorname{trace}\left\{\mathbf{Q}^{\mathrm{T}} \mathbf{W}\right\} \\
\text { subject to } & {\left[\begin{array}{cc}
\mathbf{X}_{1} & \mathbf{W} \\
\mathbf{W}^{\mathrm{T}} & \mathbf{X}_{2}
\end{array}\right] \succeq 0,} \\
& W_{i j}=W_{i j}^{\text {ref }}
\end{array}
$$

The proof follows from applying Part (a) of Theorem 7 to the above optimization. $\square$

The nuclear norm method reviewed in Subsection 1.3 corresponds to the case $\mathbf{Q}=0$ in Theorem 7.5. However, this theorem discloses the role of the weight matrix Q. In particular, this matrix can be designed based on the results developed in Section 3 to yield a small number for the upper bound given in 7.7 a), provided $\mathcal{B}$ is a sparse graph.

8. Applications. Two applications will be discussed in this section.

8.1. Optimal Power Flow Problem. Consider an $n$-bus electrical power network with the topology described by a simple graph $\mathcal{G}$, meaning that each vertex belonging to $\mathcal{V}_{\mathcal{G}}=\{1, \ldots, n\}$ represents a node of the network and each edge belonging to $\mathcal{E}_{\mathcal{G}}$ represents a transmission line. Let $y_{i j} \in \mathbb{C}$ denote the admittance of the line $(i, j) \in \mathcal{E}_{\mathcal{G}}$. Define $x \in \mathbb{C}^{n}$ as the voltage phasor vector, i.e., $x_{k}$ is the voltage phasor for node $k \in \mathcal{V}_{\mathcal{G}}$. Let $p+q$ i represent the nodal complex power vector, where $p \in \mathbb{R}^{n}$ and $q \in \mathbb{R}^{n}$ are the vectors of active and reactive powers injected at all buses. $p+q \mathbf{i}$ can be interpreted as the complex-power supply minus the complex-power demand at node $k$ of the network. The classical optimal power flow (OPF) problem is as follows: 


\begin{tabular}{|c|c||c|c|}
\hline System $\mathcal{G}$ & $\operatorname{tw}\{\mathcal{G}\}$ & System $\mathcal{G}$ & Bound on $\operatorname{tw}\{\mathcal{G}\}$ \\
\hline \hline IEEE 14-bus & 2 & Polish 2383wp & 23 \\
\hline IEEE 30-bus & 3 & Polish 2736sp & 23 \\
\hline $\begin{array}{c}\text { New England } \\
\text { 39-bus }\end{array}$ & 3 & Polish 2746wop & 23 \\
\hline IEEE 57-bus & 5 & Polish 3012wp & 24 \\
\hline IEEE 118-bus & 4 & Polish 3120sp & 24 \\
\hline IEEE 300-bus & 6 & Polish 3375wp & 25 \\
\hline
\end{tabular}

TABLE 8.1

Upper bound on the treewidth of various power systems with $n$ ranging from 14 to 3375 (the topologies of these systems can be found in MATPOWER [51]).

$$
\begin{array}{llr}
\underset{x, p, q \in \mathbb{R}^{n}}{\operatorname{minimize}} & \sum_{k \in \mathcal{V}_{\mathcal{G}}} f_{k}\left(p_{k}\right) & \\
\text { subject to } & x_{k}^{\min } \leq\left|x_{k}\right| \leq x_{k}^{\max }, & k \in \mathcal{V}_{\mathcal{G}} \\
& p_{k}^{\min } \leq p_{k} \leq p_{k}^{\max }, & k \in \mathcal{V}_{\mathcal{G}} \\
& q_{k}^{\min } \leq q_{k} \leq q_{k}^{\max }, & k \in \mathcal{V}_{\mathcal{G}} \\
& \operatorname{Re}\left\{x_{i}\left(x_{i}^{*}-x_{j}^{*}\right) y_{i j}^{*}\right\} \leq p_{i j}^{\max }, & (i, j) \in \mathcal{E}_{\mathcal{G}} \\
& p_{k}+q_{k} \mathbf{i}=\sum_{i \in \mathcal{N}_{\mathcal{G}}(k)} x_{k}\left(x_{k}^{*}-x_{i}^{*}\right) y_{k i}^{*}, & k \in \mathcal{V}_{\mathcal{G}}
\end{array}
$$

where $x_{k}^{\min }, x_{k}^{\max }, p_{k}^{\min }, p_{k}^{\max }, q_{k}^{\min }, q_{k}^{\max }$, and $p_{i j}^{\max }$ are given network limitations, and $f_{k}\left(p_{k}\right)$ is a convex function accounting for the power generation cost at node $k$. The details of this formulation may be found in 37 .

The OPF problem is a highly non-convex problem that is known to be difficult to solve in general. However, the constraints of problem 8.1) can all be expressed as linear functions of the entries of the quadratic matrix $x x^{*}$. This implies that the constraints of OPF are linear in terms of a matrix variable $\mathbf{X} \triangleq x x^{*}$. One can reformulate OPF by replacing each $x_{i} x_{j}^{*}$ by $X_{i j}$ and represent the constraints in the form of $4.5 \mathrm{a}$ ) with a union graph that is isomorphic to the network topology graph $\mathcal{G}$. In order to preserve the equivalence of the two formulations, two additional constraints must be added to the problem: (i) $\mathbf{X} \succeq 0$, (ii) $\operatorname{rank}\{\mathbf{X}\}=1$. If we drop the rank condition as the only non-convex constraint of the reformulated OPF problem, we attain the SDP relaxation of OPF that is convex. On the other hand, the parameter $\operatorname{tw}(\mathcal{G})$ is perceived to be small for graphs that describe a practical network topology. We have verified the treewidth of $\mathcal{G}$ for several power systems and reported our findings in Table 8.1. It can be seen that the treewidth of a Polish network with 3375 nodes is at most 25. Figure 8.1 shows a minimal tree decomposition associated with the IEEE 14 case. As long as the treewidth is relatively small, Theorem 4.2 states that the convex relaxation method yields a low-rank solution for the OPF problem that can be found using convex optimization.

In what follows, we will offer two case studies on the OPF problem for IEEE 300bus and Polish 2383-bus systems. For the topology graph of the IEEE 300-bus system, an optimal tree decomposition $\mathcal{T}$ of width 6 can be obtained using the exact QuickBB method in [52. Let $\mathbf{X}^{\mathrm{opt}}$ denote a solution of the SDP relaxation of optimization 8.1) for this network. Consider all submatrices of the matrix $\mathbf{X}^{\text {opt }}$ induced by the bags of 

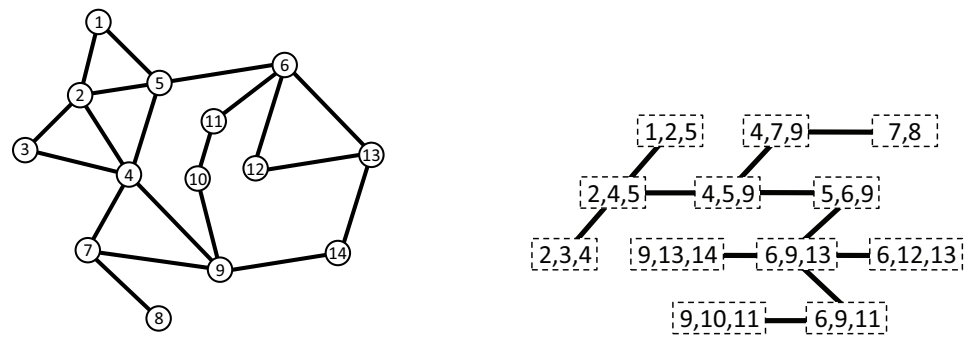

FIG. 8.1. The IEEE 14-bus test case (left figure) and its minimal tree decomposition (right figure)

$\mathcal{T}$, i.e., $\mathbf{X}^{\mathrm{opt}}\left(V_{i}\right)$ for $i=1, \ldots,|\mathcal{T}|$. Two of these submatrices have rank 2 (associated with two bags named $V_{1}$ and $V_{2}$ ) and the remaining ones have rank 1. In addition, $\left|V_{1}\right|=\left|V_{2}\right|=2$. This implies that the SDP relaxation has a rank-2 solution that can be obtained from $\mathbf{X}^{\mathrm{opt}}$ via the matrix completion technique proposed earlier, and in addition the bags $V_{1}$ and $V_{2}$ may not allow the existence of a rank-1 SDP solution. However, the off-diagonal entries of these rank-2 submatrices correspond to only two lines of the network. This implies that 2 lines of the IEEE 300-bus system make the SDP relaxation fail. To enforce the existence of a rank-1 SDP solution, we can design a penalized SDP relaxation where the loss over these problematic lines is added to the objective of the original SDP problem as a regularization term. For a suitable choice of the regularization coefficient, the penalized SDP has a rank-1 solution from which a feasible solution of OPF can be found. Define the global optimality degree associated with a feasible solution as

$$
\text { Global optimality degree }(\%)=100-\frac{\text { upper bound }- \text { lower bound }}{\text { |upper bound } \mid} \times 100
$$

where "upper bound" and "lower bound" denote the costs of the obtained feasible solution and the optimal objective value of the SDP relaxation, respectively. This measure shows the maximum distance of the suboptimal cost associated with the available feasible solution from the unknown globally minimum cost. By comparing the cost of this feasible solution to the lower bound obtained from the original SDP relaxation, the global optimality degree of the obtained feasible solution of OPF turns out to be at least 99.998\%. For the Polish 2383-bus system, the SDP relaxation has a high-rank solution, but only 5 submatrices induced by the bags of its tree decomposition are not rank-1. These so-called problematic bags contain 9 lines of the power network in total. By solving a penalized SDP relaxation with a regularization term designed based on the problematic lines, a feasible solution of OPF with the global optimality degree of at least $99 \%$ can be obtained. The details of the above simulations may be found in [53].

8.2. Optimal Distributed Control Problem. Consider the discrete-time system

$$
\left\{\begin{aligned}
x[\tau+1] & =\mathbf{A} x[\tau]+\mathbf{B} u[\tau] \quad \tau=0,1,2, \ldots \\
y[\tau] & =\mathbf{C} x[\tau]
\end{aligned}\right.
$$

with the known matrices $\mathbf{A} \in \mathbb{R}^{n \times n}, \mathbf{B} \in \mathbb{R}^{n \times m}, \mathbf{C} \in \mathbb{R}^{r \times n}$ and $x[0] \in \mathbb{R}^{n}$, where $x[\tau]$, $u[\tau]$ and $y[\tau]$ represent the state, input and output of the system, respectively. The goal is to design a decentralized (distributed) static controller minimizing a quadratic 
cost functional. Denote the controller as $u[\tau]=\mathbf{K} y[\tau]$, where the unknown controller gain $\mathbf{K}$ must belong to a given linear subspace $\mathcal{K} \subseteq \mathbb{R}^{m \times r}$. The set $\mathcal{K}$ captures the sparsity structure of the unknown decentralized controller $u[\tau]=\mathbf{K} y[\tau]$ and, more specifically, it contains all $m \times r$ real-valued matrices with forced zeros in certain entries. The optimal decentralized problem (ODC) aims to design a static controller $u[\tau]=\mathbf{K} y[\tau]$ to minimize the finite-horizon cost functional

$$
\sum_{\tau=0}^{p}\left(x[\tau]^{\mathrm{T}} \mathbf{Q} x[\tau]+u[\tau]^{\mathrm{T}} \mathbf{R} u[\tau]\right)+\gamma \operatorname{trace}\left\{\mathbf{K K}^{\mathrm{T}}\right\}
$$

subject to the system dynamics 8.2 and the controller requirement $\mathbf{K} \in \mathcal{K}$, given positive-definite matrices $\mathbf{Q}$ and $\mathbf{R}$, the coefficient $\gamma$ and the terminal time $p$. To simplify this NP-hard problem, define the vectors

$$
\begin{aligned}
& x=\left[x[0]^{\mathrm{T}} \cdots x[p]^{\mathrm{T}}\right]^{\mathrm{T}}, \quad u=\left[u[0]^{\mathrm{T}} \cdots u[p]^{\mathrm{T}}\right]^{\mathrm{T}},
\end{aligned}
$$

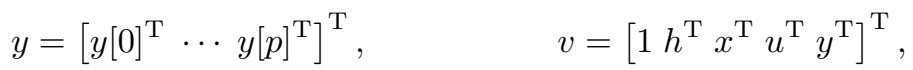

where $h$ denotes the vector of all nonzero (free) entries of $\mathbf{K}$. The objective function and constraints of the ODC problem are all quadratic with respect to the vector $v$. However, they can be cast as linear functions of the entries of the matrix $v v^{\mathrm{T}}$. Thus, by replacing $v v^{T}$ with a new variable $\mathbf{X}$, ODC can be expressed as a linear program with respect to this new variable. Nevertheless, in order to preserve the equivalence through reformulation, three additional constraints need to be imposed: (i) $\mathbf{X} \succeq 0$, (ii) $\operatorname{rank}\{\mathbf{X}\}=1$, and (iii) $X_{11}=1$. Note that the constraint (ii) carries all the non-convexity of the reformulated ODC problem. By dropping this rank constraint, an SDP relaxation of the ODC problem will be attained.

The ODC problem has a natural sparsity, which makes its SDP relaxation possess a low-rank solution. To pinpoint the underlying sparsity pattern of the problem, we construct a graph $\mathcal{G}$ as follows:

- Let $\eta$ denote the size of the vector $v$. The graph $\mathcal{G}$ has $\eta$ vertices corresponding to the entries of $v$. In particular, the vertex set of $\mathcal{G}$ can be partitioned into five vertex subsets, where subset 1 consist of a single vertex associated with the number 1 in the vector $v$ and subsets 2-5 correspond to the vectors $x, u$, $y$, and $h$, respectively.

- Given two distinct numbers $i, j \in\{1, \ldots, \eta\}$, vertices $i$ and $j$ are connected in the graph $\mathcal{G}$ if and only if the quadratic term $v_{i} v_{j}$ appears in the objective or one of the constraints of the reformulated ODC problem. As an example, vertex 1 is connected to the vertex subsets corresponding to the vectors $x$ and $u$. This is due to the fact that the linear terms $x[\tau]$ and $u[\tau]$ appear in the optimization (notice that $x[\tau]$ can be regarded as $1 \times x[\tau]$ implying the product of 1 and $x[\tau])$.

The graph $\mathcal{G}$ is highly sparse. For instance, the vertex subsets of this graph corresponding to the vectors $x$ and $u$ are isolated with no edges among them. To elucidate this property, consider the decentralized control problem for which the matrix $\mathbf{K}$ is required to be diagonal. Assume also that $\mathbf{Q}$ and $\mathbf{R}$ are diagonal. Under this circumstance, the graph $\mathcal{G}$ is depicted in Figure 8.2. To maximize the legibility of the figure, all edges of vertex 1 are not shown. Notice that after excluding vertex 1 from $\mathcal{G}$, the graph collapses to a collection of isolated vertices and stars. Hence, the parameter $\operatorname{tw}(\mathcal{G})$ is equal to 2 for the above graph. It follows from Theorem 4.2 that 




FIG. 8.2. The sparsity graph of the ODC problem in the decentralized case (some edges connected to vertex 1 are not shown to improve the readability of the graph).

the SDP relaxation of the ODC problem has a solution $\mathbf{X}^{\text {opt }}$ with rank at most 3. To obtain such a solution, we create a supergraph $\overline{\mathcal{G}}$ of $\mathcal{G}$ using Theorem 3.5 as follows. First, we connect the vertices corresponding to the $i^{\text {th }}$ and $(i+1)^{\text {th }}$ entries of $h$ for $i=1,2, \ldots, m-1$. Then, we add a new vertex to the resulting graph and connect it to all of the existing vertices. It can be shown that $|\overline{\mathcal{G}}|-\operatorname{OS}\left(\mathcal{G}_{\mathrm{s}}\right) \leq 3$ for every $\mathcal{G}_{s}$ such that $(\overline{\mathcal{G}} 入 \mathcal{G}) \subseteq \mathcal{G}_{s} \subseteq \overline{\mathcal{G}}$. Now, the supergraph $\overline{\mathcal{G}}$ can be fed into Theorem 4.2 to find a solution $\mathbf{X}^{\text {opt }}$ with rank at most 3 .

Consider now the general case where $\mathbf{Q}, \mathbf{R}$, and $\mathbf{K}$ are not necessarily diagonal. As can be seen in Figure 8.2, there is no edge in the subgraph of $\mathcal{G}$ corresponding to the entries of $x$, as long as $\mathbf{Q}$ is diagonal. However, if $\mathbf{Q}$ has nonzero off-diagonal elements, certain edges (and probably cycles) may be created in the subgraph of $\mathcal{G}$ associated with the aggregate state $x$. Under this circumstance, the treewidth of $\mathcal{G}$ could be much higher than 2 . The same argument holds for a non-diagonal $\mathbf{R}$. To understand the effect of a non-diagonal controller $\mathbf{K}$, consider the case $m=r=2$ and assume that the controller $\mathbf{K}$ under design has three free elements as follows:

$$
\mathbf{K}=\left[\begin{array}{cc}
K_{11} & K_{12} \\
0 & K_{22}
\end{array}\right]
$$

(i.e., $h_{1}=K_{11}, h_{2}=K_{12}$ and $h_{3}=K_{22}$ ). Figure 8.3 shows a part of the graph $\mathcal{G}$. It can be observed that this subgraph is acyclic for $K_{12}=0$ but has a cycle as soon as $K_{12}$ becomes a free parameter. As a result, the treewidth of $\mathcal{G}$ is contingent upon the zero pattern of $\mathbf{K}$. To deal with this issue, the ODC formulation can be diagonalized in such a way that its SDP relaxation will have a rank 1, 2 or 3 solution [54. We have performed several thousand simulations in [55] and verified that penalized SDP can be used to design distributed controllers with global optimality degrees as high as $99 \%$ for physical systems.

9. Conclusions. This paper aims to find low-rank solutions of sparse linear matrix inequality (LMI) problems using convex optimization and graph theory. To this end, the sparsity of a given LMI problem is mapped into a graph and a rigorous theory is developed to connect the rank of the minimum-rank solution of the LMI problem 

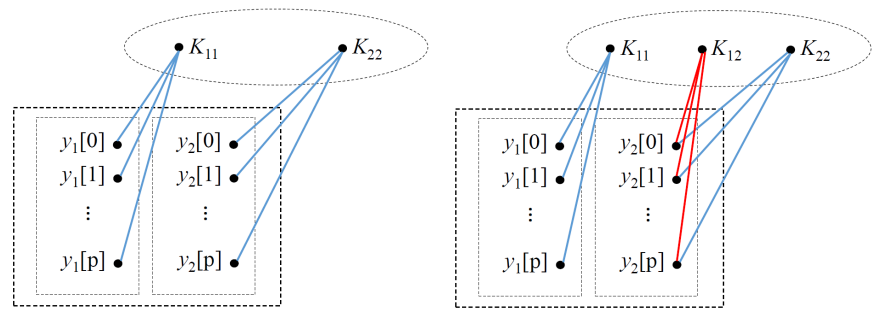

FIG. 8.3. Effect of a nonzero off-diagonal entry of the controller $\mathbf{K}:$ (a) a subgraph of $\mathcal{G}$ for the case where $K_{11}$ and $K_{22}$ are the only free parameters of the controller $\mathbf{K}$, (b) a subgraph of $\mathcal{G}$ for the case where $K_{12}$ is also a free parameter of the controller.

to the sparsity of this graph. Moreover, three graph-theoretic convex programs are proposed to find low-rank solutions of the underlying LMI problem with the property that the rank of every solution of these problems has a guaranteed upper bound. Two of these convex optimization problems may need heavy graph computation, whereas the third convex program does not rely on any computationally-expensive graph analysis and is always polynomial-time solvable. The implications of this work are also discussed for three applications: minimum-rank matrix completion, conic relaxation for polynomial optimization, and affine rank minimization. Finally, the results are applied to two case studies for electrical power networks and dynamical systems.

Acknowledgment. The authors would like to thank Professors Daniel Bienstock and Vineet Goyal of Columbia University for several fruitful discussions.

\section{REFERENCES}

[1] R. D. Monteiro, "Primal-dual path-following algorithms for semidefinite programming," SIAM Journal on Optimization, vol. 7, no. 3, pp. 663-678, 1997.

[2] Y. H. Au-Yeung and Y. T. Poon, "A remark on the convexity and positive definiteness concerning Hermitian matrices," Southeast Asian Bulletin of Mathematics, vol. 3, no. 2, pp. 85-92, 1979.

[3] A. Barvinok, "A remark on the rank of positive semidefinite matrices subject to affine constraints," Discrete \& Computational Geometry, vol. 25, no. 1, pp. 23-31, 2001.

[4] W. Ai, Y. Huang, and S. Zhang, "On the low rank solutions for linear matrix inequalities," Mathematics of Operations Research, vol. 33, no. 4, pp. 965-975, 2008.

[5] S. Sojoudi and J. Lavaei, "Exactness of semidefinite relaxations for nonlinear optimization problems with underlying graph structure," SIAM Journal on Optimization, vol. 24, no. 4, pp. 1746-1778, 2014.

[6] J. Lavaei, "Optimal decentralized control problem as a rank-constrained optimization," in Proceedings of the Allerton Conference on Control and Computing, 2013.

[7] R. Grone, C. R. Johnson, E. M. Sá, and H. Wolkowicz, "Positive definite completions of partial Hermitian matrices," Linear algebra and its applications, vol. 58, pp. 109-124, 1984.

[8] M. Fukuda, M. Kojima, K. Murota, and K. Nakata, "Exploiting sparsity in semidefinite programming via matrix completion I: General framework," SIAM Journal on Optimization, vol. 11, no. 3, pp. 647-674, 2001.

[9] K. Nakata, K. Fujisawa, M. Fukuda, M. Kojima, and K. Murota, "Exploiting sparsity in semidefinite programming via matrix completion II: Implementation and numerical results," Mathematical Programming, vol. 95, no. 2, pp. 303-327, 2003.

[10] S. Kim, M. Kojima, M. Mevissen, and M. Yamashita, "Exploiting sparsity in linear and nonlinear matrix inequalities via positive semidefinite matrix completion," Mathematical programming, vol. 129, no. 1, pp. 33-68, 2011.

[11] R. Jabr, "Exploiting sparsity in SDP relaxations of the OPF problem," IEEE Transactions on Power Systems,, vol. 27, no. 2, pp. 1138-1139, 2012.

[12] D. K. Molzahn, J. T. Holzer, B. C. Lesieutre, and C. L. DeMarco, "Implementation of a large- 
scale optimal power flow solver based on semidefinite programming," IEEE Transactions on Power Systems, vol. 28, no. 4, pp. 3987-3998, 2013.

[13] M. S. Andersen, A. Hansson, and L. Vandenberghe, "Reduced-complexity semidefinite relaxations of optimal power flow problems," IEEE Transactions on Power Systems, vol. 29, no. 4, pp. 1855-1863, July 2014.

[14] M. Laurent and A. Varvitsiotis, "A new graph parameter related to bounded rank positive semidefinite matrix completions," Mathematical Programming, vol. 145, no. 1-2, pp. 291$325,2014$.

[15] S. M. Fallat and L. Hogben, "The minimum rank of symmetric matrices described by a graph: a survey," Linear Algebra and its Applications, vol. 426, no. 2, pp. 558-582, 2007.

[16] W. Barrett, H. van der Holst, and R. Loewy, "Graphs whose minimal rank is two," Electronic Journal of Linear Algebra, vol. 11, no. 258-280, p. 687, 2004.

[17] J. Sinkovic and H. van der Holst, "The minimum semidefinite rank of the complement of partial k-trees," Linear Algebra and its Applications, vol. 434, no. 6, pp. 1468-1474, 2011.

[18] F. Barioli, W. Barrett, S. M. Fallat, H. T. Hall, L. Hogben, B. Shader, P. Van Den Driessche, and H. van der Holst, "Zero forcing parameters and minimum rank problems," Lin. Alg. Appl., vol. 433, no. 2, pp. 401-411, 2010.

[19] P. Hackney, B. Harris, M. Lay, L. H. Mitchell, S. K. Narayan, and A. Pascoe, "Linearly independent vertices and minimum semidefinite rank," Linear Algebra and its Applications, vol. 431, no. 8, pp. 1105-1115, 2009.

[20] L. H. Mitchell, S. K. Narayan, and A. M. Zimmer, "Lower bounds in minimum rank problems," Lin. Alg. Appl., vol. 432, no. 1, pp. 430-440, 2010.

[21] F. Barioli, W. Barrett, S. M. Fallat, H. T. Hall, L. Hogben, B. Shader, P. van den Driessche, and H. Van Der Holst, "Parameters related to tree-width, zero forcing, and maximum nullity of a graph," J. Graph Theory, vol. 72, no. 2, pp. 146-177, 2013.

[22] S. Fallat and L. Hogben, "Minimum rank, maximum nullity, and zero forcing number of graphs," Handbook of Linear Algebra, 2nd edition, L. Hogben editor, CRC Press, Boca Raton, 2013.

[23] S. Arnborg, D. G. Corneil, and A. Proskurowski, "Complexity of finding embeddings in a k-tree," SIAM Journal on Algebraic Discrete Methods, vol. 8, no. 2, pp. 277-284, 1987.

[24] J. Matoušek and R. Thomas, "Algorithms finding tree-decompositions of graphs," Journal of Algorithms, vol. 12, no. 1, pp. 1-22, 1991.

[25] H. L. Bodlaender, "A linear-time algorithm for finding tree-decompositions of small treewidth," SIAM Journal on computing, vol. 25, no. 6, pp. 1305-1317, 1996.

[26] H. L. Bodlaender and A. M. Koster, "Treewidth computations I. upper bounds," Inform. Comput., vol. 208, no. 3, pp. 259-275, 2010.

[27] — - "Treewidth computations II. lower bounds," Inform. Comput., vol. 209, no. 7, pp. 11031119, 2011.

[28] M. Booth, P. Hackney, B. Harris, C. R. Johnson, M. Lay, L. H. Mitchell, S. K. Narayan, A. Pascoe, K. Steinmetz, B. D. Sutton et al., "On the minimum rank among positive semidefinite matrices with a given graph," SIAM Journal on Matrix Analysis and Applications, vol. 30, no. 2, pp. 731-740, 2008.

[29] N. Shor, "Quadratic optimization problems," Soviet Journal of Circuits and Systems Sciences, vol. 25 , no. $1-11$, p. $6,1987$.

[30] Y. Nesterov, A. S. Nemirovskii, and Y. Ye, Interior-point polynomial algorithms in convex programming. SIAM, 1994, vol. 13.

[31] L. Vandenberghe and S. Boyd, "Semidefinite programming," SIAM review, vol. 38, no. 1, pp. 49-95, 1996.

[32] K. M. Anstreicher, "On convex relaxations for quadratically constrained quadratic programming," Mathematical programming, vol. 136, no. 2, pp. 233-251, 2012.

[33] M. X. Goemans and D. P. Williamson, "Improved approximation algorithms for maximum cut and satisfiability problems using semidefinite programming," Journal of the ACM (JACM), vol. 42, no. 6, pp. 1115-1145, 1995.

[34] J. Briet, F. M. de Oliveira Filho, and F. Vallentin, "Grothendieck inequalities for semidefinite programs with rank constraint," Theory of Computing, vol. 10, no. 4, pp. 77-105, 2010.

[35] N. Alon, K. Makarychev, Y. Makarychev, and A. Naor, "Quadratic forms on graphs," Inventiones mathematicae, vol. 163, no. 3, pp. 499-522, 2006.

[36] M. Laurent and A. Varvitsiotis, "Computing the Grothendieck constant of some graph classes," Operations Research Letters, vol. 39, no. 6, pp. 452-456, 2011.

[37] J. Lavaei and S. H. Low, "Zero duality gap in optimal power flow problem," IEEE Transactions on Power Systems, vol. 27, no. 1, pp. 92-107, 2012.

[38] J. B. Lasserre, "An explicit exact SDP relaxation for nonlinear 0-1 programs," in Integer Programming and Combinatorial Optimization. Springer, 2001, pp. 293-303. 
[39] S. Kim and M. Kojima, "Exact solutions of some nonconvex quadratic optimization problems via SDP and SOCP relaxations," Computational Optimization and Applications, vol. 26, no. 2, pp. 143-154, 2003.

[40] J. Lavaei, A. Babakhani, A. Hajimiri, and J. C. Doyle, "Solving large-scale hybrid circuitantenna problems," IEEE Transactions on Circuits and Systems I: Regular Papers, vol. 58, no. 2, pp. 374-387, 2011.

[41] R. Madani, S. Sojoudi, and J. Lavaei, "Convex relaxation for optimal power flow problem: Mesh networks," IEEE Transactions on Power Systems, vol. 30, no. 1, pp. 199-211, Jan 2015.

[42] B. Recht, M. Fazel, and P. A. Parrilo, "Guaranteed minimum-rank solutions of linear matrix equations via nuclear norm minimization," SIAM review, vol. 52, no. 3, pp. 471-501, 2010.

[43] M. Fazel, "Matrix rank minimization with applications," Ph.D. dissertation, Stanford University, 2002.

[44] C. R. Johnson, "Matrix completion problems: a survey," in Proceedings of Symposia in Applied Mathematics, vol. 40, 1990, pp. 171-198.

[45] E. J. Candès and B. Recht, "Exact matrix completion via convex optimization," Foundations of Computational mathematics, vol. 9, no. 6, pp. 717-772, 2009.

[46] R. H. Keshavan, A. Montanari, and S. Oh, "Matrix completion from a few entries," Information Theory, IEEE Transactions on, vol. 56, no. 6, pp. 2980-2998, 2010.

[47] E. J. Candès and T. Tao, "The power of convex relaxation: Near-optimal matrix completion," IEEE Transactions on Information Theory, vol. 56, no. 5, pp. 2053-2080, 2010.

[48] B. Recht, W. Xu, and B. Hassibi, "Null space conditions and thresholds for rank minimization," Mathematical programming, vol. 127, no. 1, pp. 175-202, 2011.

[49] M. Fazel, H. Hindi, and S. P. Boyd, "Log-det heuristic for matrix rank minimization with applications to Hankel and Euclidean distance matrices," in American Control Conference, 2003. Proceedings of the 2003, vol. 3. IEEE, 2003, pp. 2156-2162.

[50] D. Carlson, E. Haynsworth, and T. Markham, "A generalization of the Schur complement by means of the Moore-Penrose inverse," SIAM Journal on Applied Mathematics, vol. 26, no. 1 , pp. $169-175,1974$.

[51] R. D. Zimmerman, C. E. Murillo-Sánchez, and R. J. Thomas, "MATPOWER's extensible optimal power flow architecture," in IEEE Power and Energy Society General Meeting, 2009.

[52] V. Gogate and R. Dechter, "A complete anytime algorithm for treewidth," in Proceedings of the 20th conference on Uncertainty in artificial intelligence. AUAI Press, 2004, pp. 201-208.

[53] R. Madani, M. Ashraphijuo, and J. Lavaei, "Promises of conic relaxation for contingencyconstrained optimal power flow problem," IEEE Transactions on Power Systems, vol. 31, no. 2, pp. 1297-1307, March 2016.

[54] G. Fazelnia, R. Madani, and J. Lavaei, "Convex relaxation for optimal distributed control problems," IEEE Transactions on Automatic Control, vol. 62, no. 1, pp. 206-221, Jan 2017.

[55] A. Kalbat, R. Madani, G. Fazelnia, and J. Lavaei, "Efficient convex relaxation for stochastic optimal distributed control problem," in 52nd Annual Allerton Conference on Communication, Control, and Computing (Allerton), Sept 2014, pp. 589-596. 